\title{
Quasiconvex Envelopes in Nonlinear Elasticity
}

\author{
Annie Raoult \\ Laboratoire MAP5, Université Paris Descartes, Paris, France \\ annie.raoult@parisdescartes.fr
}

\begin{abstract}
We give several examples of modeling in nonlinear elasticity where a quasiconvexification procedure is needed. We first recall that the three-dimensional Saint Venant-Kirchhoff energy fails to be quasiconvex and that its quasiconvex envelope can be obtained by means of careful computations. Second, we turn to the mathematical derivation of slender structure models: an asymptotic procedure using $\Gamma$-convergence tools leads to models whose energy is quasiconvex by construction. Third, we construct an homogenized quasiconvex energy for square lattices.
\end{abstract}

\section{The Saint Venant-Kirchhoff stored energy function}

\subsection{Non quasiconvexity of the Saint Venant-Kirchhoff stored en- ergy function}

This section is based on Raoult (1986) from which it is immediately derived that the Saint Venant-Kirchhoff stored energy function is not rankone convex, and as a consequence not polyconvex, nor quasiconvex.

The internal energy of an elastic material reads $J(\phi)=\int_{\Omega} W(\nabla \phi(x)) d x$ where $\Omega \subset \mathbb{R}^{3}$ is a reference configuration (here assumed to be homogeneous), $W: \mathbb{M}_{3 \times 3} \mapsto \mathbb{R}$ is the stored energy function that is most of the time assumed to be continuous and the deformation $\phi: \Omega \mapsto \mathbb{R}^{3}$ is sufficiently regular. This is the energy due to the deformation $\phi$. Actually, the domain of $W$ should be restricted to the set $\mathbb{M}_{3 \times 3}^{+}$of matrices with positive determinant and $\phi$ should satisfy in some sense $\operatorname{det} \nabla \phi(x)>0$ in order to express that orientation is preserved by realistic deformations and to prevent matter interpenetration. This restriction leads to mathematical difficulties and is quite often left aside. The total energy is the sum of the internal energy and of the external energy which takes into account the action of external loads (body forces such as gravity, surface forces such as 
pressure ...). The equilibrium problem in an energy form reads:

$$
\text { Find } \phi \in \Phi \text { such that } I(\phi)=\min _{\Phi} I
$$

where $I$ is the total energy and $\Phi$ is a functional space that takes into account placement conditions. A basic hypothesis for proving the existence of a minimizer of a given functional is that this functional be lower semicontinuous. We are dealing here with functionals defined on infinite dimensional spaces, namely $W^{1, p}\left(\Omega ; \mathbb{R}^{3}\right)$ spaces (for simple growth conditions on $W, J$ is well defined on $\left.W^{1, p}\left(\Omega ; \mathbb{R}^{3}\right)\right)$. Therefore, the appropriate lower semicontinuity is lower semicontinuity with respect to the weak topology. Then, it is classical (see Morrey (1995) and Dacorogna (2007) for a survey) that $J$ is weakly lower semicontinuous if and only if $W$ is quasiconvex. Recall that the quasiconvexity condition reads

$$
\forall F \in \mathbb{M}_{3 \times 3}, \forall \phi \in W_{0}^{1, \infty}\left(\Omega ; \mathbb{R}^{3}\right), W(F) \leq \frac{1}{|\Omega|} \int_{\Omega} W(F+\nabla \phi(x)) d x .
$$

Quasiconvexity is a nonlocal notion which makes it difficult to check or to contradict. Two pointwise notions make a lower-upper frame for quasiconvexity. Indeed, the implications

$$
\text { polyconvexity } \Rightarrow \text { quasiconvexity } \Rightarrow \text { rank-one convexity }
$$

are valid. This chain of implications can be easily remembered by noticing that the three notions are listed in alphabetical order. The convexity notion can even be added at the left extremity of the chain. But for applications in nonlinear elasticity, this is not useful since convexity of the energy density has to be ruled out for modeling reasons. Rank-one convexity is simply convexity along the straight line generated by two matrices whose difference is of rank 1. It reads

$$
\begin{aligned}
& \forall F, G \in \mathbb{M}_{3,3} \text { such that } \operatorname{rank}(F-G) \leq 1, \\
& \forall \lambda \in[0,1], W(\lambda F+(1-\lambda) G) \leq \lambda W(F)+(1-\lambda) W(G) .
\end{aligned}
$$

Let us mention that rank-one convexity does not imply quasiconvexity as was proved by Sverak (1992). Polyconvexity is a more complex notion that was introduced in Ball (1977): an energy is polyconvex if one can find a convex function $w$ such that

$$
\forall F \in \mathbb{M}_{3 \times 3}, W(F)=w(F, \operatorname{adj} F, \operatorname{det} F) .
$$

The function $w$ in the above equation is defined on $\mathbb{M}_{3 \times 3} \times \mathbb{M}_{3 \times 3} \times \mathbb{R}$. Note that this definition may be restricted to matrices with positive determinant 
in which case $w$ is defined on $\mathbb{M}_{3 \times 3} \times \mathbb{M}_{3 \times 3} \times \mathbb{R}^{+*}$ which is the convex hull of $\{(F, \operatorname{adj} F, \operatorname{det} F), \operatorname{det} F>0\}$. From implications (3), it is obvious that a way of proving that an energy is quasiconvex - the important notion - is to prove that it is polyconvex. This is why the possible polyconvexity of the Saint Venant-Kirchhoff energy was examined two decades ago. In fact, the proof provided the non rank-one convexity.

Theorem 1.1. The Saint Venant-Kirchhoff energy is not rank-one convex.

The following statements immediately follow.

Corollary 1.2. The Saint Venant-Kirchhoff energy is not polyconvex, nor quasiconvex.

Proof of Theorem 1.1. - The Saint Venant Kirchhoff energy reads

$$
W(F)=\frac{\mu}{4}\left\|F^{T} F-I\right\|^{2}+\frac{\lambda}{8}\left(\|F\|^{2}-3\right)^{2},
$$

where $\|F\|^{2}=\operatorname{tr} F^{T} F$. Letting $C(F)=F^{T} F$, we have equivalently

$$
W(F)=\frac{\mu}{4} \operatorname{tr}(C(F))^{2}+\frac{\lambda}{8}\|F\|^{4}-\frac{3 \lambda+2 \mu}{4}\|F\|^{2}+\frac{9 \lambda+6 \mu}{8} .
$$

The first term reads $\frac{\mu}{4}\left(v_{1}^{4}+v_{2}^{4}+v_{3}^{4}\right)$ where $v_{1}, v_{2}, v_{3}$ are the singular values of $F$. Since $g$ defined by $g\left(v_{1}, v_{2}, v_{3}\right)=\left(v_{1}^{4}+v_{2}^{4}+v_{3}^{4}\right)$ is a convex, symmetric function that is not decreasing with respect to each of its variable, it is known that this first term is convex in $F$. Proofs of such results can be found in Ball (1977), Ciarlet (1987), Le Dret (1990), Thompson and Freede (1971). The second term is obviously convex in $F$. But, the minus sign in the third term prevents the whole of the expression of being rank-one convex as shown by the following counter-example which uses the fact that for $\|F\|$ small, this term is greater than the two previous ones. Let $F=\varepsilon I d$ and $G=\varepsilon \operatorname{diag}(1,1,3)$, so that $\frac{F+G}{2}=\varepsilon \operatorname{diag}(1,1,2)$. Notice that $F-G$ is of rank 1. Matrices $F$ and $G$ are such that

$$
\operatorname{adj}\left(\frac{F+G}{2}\right)=\frac{\operatorname{adj} F+\operatorname{adj} G}{2}, \operatorname{det}\left(\frac{F+G}{2}\right)=\frac{\operatorname{det} F+\operatorname{det} G}{2} .
$$

If $W$ were rank-one convex, we would get $W\left(\frac{F+G}{2}\right) \leq \frac{1}{2}(W(F)+W(G))$, i.e.,

$$
\begin{aligned}
& \mu \operatorname{tr}\left(C\left(\frac{F+G}{2}\right)\right)^{2}+\frac{\lambda}{2}\left\|\frac{F+G}{2}\right\|^{4}-(3 \lambda+2 \mu)\left\|\frac{F+G}{2}\right\|^{2} \\
& \leq \frac{1}{2}\left[\mu \operatorname{tr}(C(F))^{2}+\frac{\lambda}{2}\|F\|^{4}-(3 \lambda+2 \mu)\|F\|^{2}\right] \\
& +\frac{1}{2}\left[\mu \operatorname{tr}(C(G))^{2}+\frac{\lambda}{2}\|G\|^{4}-(3 \lambda+2 \mu)\|G\|^{2}\right] .
\end{aligned}
$$


Since terms $\operatorname{tr}(C(F))^{2}, \operatorname{tr}(C(G))^{2}, \operatorname{tr}\left(C\left(\frac{F+G}{2}\right)\right)^{2}$ and terms $\|F\|^{4},\|G\|^{4}$, $\left\|\frac{F+G}{2}\right\|^{4}$ are of order 4 with respect to $\varepsilon$ while the remaining ones are of order 2 , this amounts to

$$
-\frac{1}{\varepsilon^{2}}\left\|\frac{F+G}{2}\right\|^{2} \leq-\frac{1}{\varepsilon^{2}}\left(\frac{1}{2}\|F\|^{2}+\frac{1}{2}\|G\|^{2}\right) .
$$

The contradiction follows from the equalities $\|F\|^{2}=3 \varepsilon^{2},\|G\|^{2}=11 \varepsilon^{2}$, $\left\|\frac{F+G}{2}\right\|^{2}=6 \varepsilon^{2}$.

\subsection{The quasiconvex envelope of the Saint Venant-Kirchhoff stored energy function}

The quasiconvex envelope of the three-dimensional Saint Venant-Kirchhoff stored energy function was first computed in Le Dret and Raoult (1995b). Computations were made in a systematic but somewhat tedious way, and inspired by a preliminary work by the authors in a $2 d \times 3 d$ setting, see Le Dret and Raoult (1995a). Extending some results by Pipkin allows to simplify the proofs, see Pipkin (1994), Le Dret and Raoult (1995c).

We denote by $\mathbb{S}_{m}^{+}$the set of symmetric, positive semidefinite matrices.

Lemma 1.3. Let $m \leq n$ and $Y: \mathbb{M}_{n \times m} \mapsto \mathbb{R}$ be a left $O(n)$-invariant, rankone convex mapping. Then the mapping $\tilde{Y}: \mathbb{S}_{m}^{+} \mapsto \mathbb{R}$ such that $Y(F)=$ $\tilde{Y}\left(F^{T} F\right)$ for all $F$ in $\mathbb{M}_{n \times m}$ satisfies

$$
\tilde{Y}(C) \leq \tilde{Y}(C+S) \text { for all } C, S \in \mathbb{S}_{m}^{+} .
$$

Remark 1.4. In the case when $m<n$, this result is due to Pipkin (1994). However, the argument does not apply to square matrices. In Pipkin's terminology, $\tilde{Y}$ is said to be increasing.

Proof - Following Pipkin (1993), we first remark that proving (8) amounts to proving that

$$
\tilde{Y}(C) \leq \tilde{Y}(C+\mu v \otimes v) \text { for all } C \in \mathbb{S}_{m}^{+} \text {and for all } \mu \geq 0, v \in \mathbb{R}^{m} \backslash\{0\} .
$$

Indeed, (8) clearly implies (9). Conversely, any $S$ in $\mathbb{S}_{m}^{+}$admits a spectral decomposition $S=\sum_{i=1, m} \mu_{i} v_{i} \otimes v_{i}$ where $\mu_{i} \geq 0$, and $v_{i}, i=1, \cdots, m$ are orthonormal eigenvectors of $S$. Applying inequality (9) $m$ times, we obtain (8).

Let us now prove (9). Let $C \in \mathbb{S}_{m}^{+}$and $v \in \mathbb{R}^{m} \backslash\{0\}$ be given. Without loss of generality, we assume $\|v\|^{2}:=v^{T} v=1$. 
We first consider the case when either $m<n$ or $m=n$ and $C$ is not invertible. In both cases, $C$ can be written as $C=F^{T} F$ where $F^{T}$ is a noninjective $m \times n$ matrix. Therefore, there exists $u$ in $\operatorname{ker} F^{T}$ with $\|u\|=1$. From the rank-one convexity of $Y$, we know that the function $y: t \in \mathbb{R} \mapsto y(t)=Y(F+t u \otimes v) \in \mathbb{R}$ is convex. Moreover, since $F^{T} u=0$, $y(t)=\tilde{Y}\left(C+t^{2} v \otimes v\right)$. Therefore, $y$ is even. It follows that $y$ is monotone increasing on $\mathbb{R}^{+}$; in particular, $y(0) \leq y(t)$ for all $t \geq 0$. Choosing $t=\sqrt{\mu}$, we obtain (9).

We now turn to the case when $m=n$ and $C$ is invertible. For all $\mu \geq 0$, the matrix $C_{\mu}=C+\mu v \otimes v$ is symmetric, positive definite. Hence, $F_{\mu}=C_{\mu}^{1 / 2}$ is invertible. We define a function $h$ on $\mathbb{R}$ by

$$
h: t \in \mathbb{R} \mapsto h(t)=Y\left(F_{\mu}+t F_{\mu}^{-1} v \otimes v\right) .
$$

It follows from the rank-one convexity of $Y$ that $h$ is convex. Moreover,

$$
h(t)=\tilde{Y}\left(C+(\mu+2 t) v \otimes v+t^{2} v \otimes v(C+\mu v \otimes v)^{-1} v \otimes v\right) .
$$

An easy computation shows that the function

$$
t \in \mathbb{R} \mapsto 2 t v \otimes v+t^{2} v \otimes v(C+\mu v \otimes v)^{-1} v \otimes v
$$

is symmetric with respect to $\bar{t}=-\left(v^{T}(C+\mu v \otimes v)^{-1} v\right)^{-1}<0$. The function $h$, in turn, is symmetric with respect to $\bar{t}$. Therefore, $h$ attains its minimum at $\bar{t}$ and is monotone increasing on $[\bar{t},+\infty[$. Obviously, $h(0)=\tilde{Y}(C+\mu v \otimes v)$. If we can find $t$ such that $\bar{t} \leq t \leq 0$ and

$$
h(t)=\tilde{Y}(C),
$$

then inequality (9) is proved. A sufficient condition for a real number $t$ to solve $(10)$ is

$$
t^{2} v^{T}(C+\mu v \otimes v)^{-1} v+2 t+\mu=0 .
$$

The discriminant of equation (11) is positive if and only if

$$
\mu v^{T}(C+\mu v \otimes v)^{-1} v \leq 1 .
$$

Let us check that this is indeed the case. Let $z=\mu^{1 / 2} C^{-1 / 2} v$. Then we have

$$
\mu v^{T}(C+\mu v \otimes v)^{-1} v=z^{T}(I+z \otimes z)^{-1} z=\frac{\|z\|^{2}}{1+\|z\|^{2}} \leq 1,
$$

hence the roots of equation (11) are real. Moreover, they are nonpositive and symmetric with respect to $\bar{t}$. The largest root thus satisfies $\bar{t} \leq t \leq 0$ and (10), which proves our claim.

With this lemma at hand, we can now state our main result. 
Theorem 1.5. Let $m \leq n$ and let $W: F \in \mathbb{M}_{n \times m} \mapsto \mathbb{R}$ be a left $O(n)$ invariant, bounded from below, stored energy function such that the associated function $\tilde{W}: C \mapsto \tilde{W}(C)$ is convex on $\mathbb{S}_{m}^{+}$. Then,

$$
Q W(F)=\inf _{S \in \mathbb{S}_{m}^{+}} \tilde{W}\left(F^{T} F+S\right) .
$$

Proof - Since $\tilde{W}$ is bounded from below, we can define, with Pipkin's notation, a function $\tilde{W}_{r}$ on $\mathbb{S}_{m}^{+}$by $\tilde{W}_{r}(C)=\inf _{S \in \mathbb{S}_{m}^{+}} \tilde{W}(C+S)$. It is easy to check that $\tilde{W}_{r}(C)$ is convex. Indeed, given $C_{1}$ and $C_{2} \in \mathbb{S}_{m}^{+}$and an arbitrary $\varepsilon>0$, there exists $S_{1}$ and $S_{2}$ in $\mathbb{S}_{m}^{+}$such that $\tilde{W}_{r}\left(C_{i}\right) \leq \tilde{W}\left(C_{i}+S_{i}\right)$ and $\tilde{W}\left(C_{i}+S_{i}\right) \leq \tilde{W}_{r}\left(C_{i}\right)+\varepsilon$ for $i=1,2$. Let $t \in[0,1]$ and $S=t S_{1}+(1-t) S_{2}$. Then,

$$
\begin{aligned}
\tilde{W}_{r}\left(t C_{1}+(1-t) C_{2}\right) & \leq \tilde{W}\left(t C_{1}+(1-t) C_{2}+S\right) \\
& \leq t \tilde{W}\left(C_{1}+S_{1}\right)+(1-t) \tilde{W}\left(C_{2}+S_{2}\right) \\
& \leq t \tilde{W}_{r}\left(C_{1}\right)+(1-t) \tilde{W}_{r}\left(C_{2}\right)+\varepsilon
\end{aligned}
$$

The convexity of $\tilde{W}_{r}$ follows at once.

Let us now remark that $\tilde{W}_{r}$ obviously satisfies $\tilde{W}_{r}(C) \leq \tilde{W}_{r}(C+S)$ for all $C$ and $S$ in $\mathbb{S}_{m}^{+}$. This implies that the function $Z:=F \in \mathbb{M}_{n \times m} \mapsto$ $\tilde{W}_{r}\left(F^{T} F\right)$ is convex. Indeed, for all $F$ and $G$ in $\mathbb{M}_{n \times m}$ and for all $t \in[0,1]$,

$$
\begin{aligned}
(t F+(1-t) G)^{T}(t F+(1-t) G) & =t F^{T} F+(1-t) G^{T} G \\
& -t(1-t)(F-G)^{T}(F-G) .
\end{aligned}
$$

Therefore, since $t(1-t)(F-G)^{T}(F-G)$ is positive semidefinite,

$$
Z(t F+(1-t) G) \leq \tilde{W}_{r}\left(t F^{T} F+(1-t) G^{T} G\right) \leq t Z(F)+(1-t) Z(G),
$$

by the convexity of $\tilde{W}_{r}$. Consequently, since $Z$ is convex and below $W$, we see that $Z \leq Q W$.

The reverse inequality is obtained as follows. From Le Dret and Raoult (1995a), we know that $Q W$ is also left $O(n)$-invariant. Applying Lemma 1 to $Y=Q W$, which is rank-one convex, we obtain

$$
\begin{aligned}
Q W(F)=\widetilde{Q W}\left(F^{T} F\right) & \leq \widetilde{Q W}\left(F^{T} F+S\right)=Q W\left(\left(F^{T} F+S\right)^{1 / 2}\right) \\
& \leq \tilde{W}\left(F^{T} F+S\right)
\end{aligned}
$$

for all $S \in \mathbb{S}_{m}^{+}$. Therefore, $Q W(F) \leq Z(F)$ and the proof is complete. 
Remark 1.6. i) It follows clearly from the proof that the quasiconvex envelope is also in this case the convex and rank-one convex envelope of the stored energy function.

ii) We now proceed to show by means of a simple counterexample that Pipkin's formula fails for $m>n$. Let $m=2, n=1$. Consider the function $W: \mathbb{M}_{1 \times 2} \mapsto \mathbb{R}, W(F)=\left\|F^{T} F-I\right\|^{2}$. We thus let $\tilde{W}(C)=\|C-I\|^{2}$. This function is clearly convex with respect to $C$. If we denote $F=\left(z_{1}, z_{2}\right)$ with $z_{i} \in \mathbb{R}$, then we have

$$
W(F)=\left(z_{1}^{2}-1\right)^{2}+\left(z_{2}^{2}-1\right)^{2}+2 z_{1}^{2} z_{2}^{2}=\left(\|F\|^{2}-1\right)^{2}+1 .
$$

Therefore, $Q W(F)=C W(F)$ and $Q W(F)=W(F)$ if $\|F\| \geq 1,1$ if $\|F\| \leq 1$, see Dacorogna (2007). Let us now take $F=(1,-1)$ so that $C=\left(\begin{array}{cc}1 & -1 \\ -1 & 1\end{array}\right)$ and $\tilde{W}(C)=2$. With the choice $S=\frac{1}{2}\left(\begin{array}{ll}1 & 1 \\ 1 & 1\end{array}\right)$, we obtain $\tilde{W}_{r}(C) \leq \tilde{W}(C+S)=1<2=\tilde{W}(C)=W(F)=Q W(F)$.

Application to an explicit computation: The Saint Venant-Kirchhoff stored energy function defined in (6) can equivalently be written under the form $W(F)=\tilde{W}\left(F^{T} F\right)$ where

$$
\tilde{W}(C)=\frac{\mu}{4}\|C-I\|^{2}+\frac{\lambda}{8}(\operatorname{tr} C-3)^{2}
$$

for all $C$ in $\mathbb{S}_{3}^{+}$. The mapping $\tilde{W}$ is clearly convex with respect to $C$.

Therefore, Theorem 1.5 applies. Let us briefly show how computations can be organized. For any $C$ in $\mathbb{S}_{3}^{+}$, let $J_{C}: S \in \mathbb{S}_{3}^{+} \mapsto \tilde{W}(C+S) \in \mathbb{R}$. This is a strictly convex, coercive mapping. Consequently, $J_{C}$ admits one and only one minimizer on $\mathbb{S}_{3}^{+}$. By (13), we have to evaluate $\inf _{S \in \mathbb{S}_{3}^{+}} J_{C}(S)=$ $\min _{S \in \mathbb{S}_{3}^{+}} J_{C}(S)$. Assume first that $C$ is diagonal. We deduce from (14) that $J_{C}(S) \geq J_{C}\left(\operatorname{diag}\left(s_{11}, s_{22}, s_{33}\right)\right)$. Minimizing $J_{C}(S)$ among semidefinite positive matrices thus amounts to minimizing $J_{C}(S)$ among diagonal positive matrices. Equivalently, we have to minimize on $\left(\mathbb{R}^{+}\right)^{3}$ the mapping $j_{C}$ such that

$$
j_{C}\left(s_{1}, s_{2}, s_{3}\right)=\frac{\mu}{4} \sum_{i=1}^{3}\left(c_{i i}-1+s_{i}\right)^{2}+\frac{\lambda}{8}\left(\sum_{i=1}^{3}\left(c_{i i}-1+s_{i}\right)\right)^{2} .
$$

Without loss of generality, we assume that $c_{11} \leq c_{22} \leq c_{33}$. The optimality conditions for $j_{C}$ on $\left(\mathbb{R}^{+}\right)^{3}$ read

$$
\begin{gathered}
D j_{C}\left(s_{1}, s_{2}, s_{3}\right)\left(t_{1}, t_{2}, t_{3}\right) \geq 0 \text { for all }\left(t_{1}, t_{2}, t_{3}\right) \in\left(\mathbb{R}^{+}\right)^{3}, \\
D j_{C}\left(s_{1}, s_{2}, s_{3}\right)\left(s_{1}, s_{2}, s_{3}\right)=0,\left(s_{1}, s_{2}, s_{3}\right) \in\left(\mathbb{R}^{+}\right)^{3} .
\end{gathered}
$$


They are equivalent to

$$
\begin{gathered}
\partial_{i} j_{C}\left(s_{1}, s_{2}, s_{3}\right) \geq 0 \text { for } i=1,2,3, \\
\partial_{i} j_{C}\left(s_{1}, s_{2}, s_{3}\right) s_{i}=0, s_{i} \geq 0 \text { for } i=1,2,3,
\end{gathered}
$$

that is to say

$$
\begin{gathered}
(2 \mu+\lambda)\left(c_{i i}-1+s_{i}\right)+\lambda \sum_{k \neq i}\left(c_{k k}-1+s_{k}\right) \geq 0, \\
\left((2 \mu+\lambda)\left(c_{i i}-1+s_{i}\right)+\lambda \sum_{k \neq i}\left(c_{k k}-1+s_{k}\right)\right) s_{i}=0, s_{i} \geq 0 .
\end{gathered}
$$

We distinguish four different cases:

1) If $c_{33} \leq 1$, then we can choose $s_{i}=1-c_{i i}$ and $\min _{\left(\mathbb{R}^{+}\right)^{3}} j_{C}=0$.

2) If $c_{33} \geq 1$ and $2(\lambda+\mu) c_{22}+\lambda c_{33} \leq 3 \lambda+2 \mu$, then we can choose $s_{3}=0, s_{j}=-c_{j j}-\frac{\lambda}{2(\lambda+\mu)} c_{33}+\frac{3 \lambda+2 \mu}{2(\lambda+\mu)} \geq 0, j=1,2$ and

$$
\min _{\left(\mathbb{R}^{+}\right)^{3}} j_{C}=\frac{\mu(3 \lambda+2 \mu)}{8(\lambda+\mu)}\left(c_{33}-1\right)^{2} .
$$

3) If $2(\lambda+\mu) c_{22}+\lambda c_{33} \geq 3 \lambda+2 \mu$ and $(\lambda+2 \mu) c_{11}+\lambda\left(c_{22}+c_{33}\right) \leq 3 \lambda+2 \mu$, then $s_{2}=s_{3}=0, s_{1}=-c_{11}-\frac{\lambda}{(\lambda+2 \mu)}\left(c_{22}+c_{33}\right)+\frac{3 \lambda+2 \mu}{(\lambda+2 \mu)} \geq 0$ and

$$
\min _{\left(\mathbb{R}^{+}\right)^{3}} j_{C}=\frac{\mu}{4}\left(\left(c_{22}-1\right)^{2}+\left(c_{33}-1\right)^{2}\right)+\frac{\lambda \mu}{4(\lambda+2 \mu)}\left(c_{22}+c_{33}-2\right)^{2} .
$$

4) If $(\lambda+2 \mu) c_{11}+\lambda\left(c_{22}+c_{33}\right) \geq 3 \lambda+2 \mu$, then we can choose $s_{1}=s_{2}=$ $s_{3}=0$ and $\min _{\left(\mathbb{R}^{+}\right)^{3}} j_{C}=\tilde{W}(C)$.

So far, we have determined $Q W(F)$ when $C=F^{T} F$ is diagonal. To extend the result to arbitrary matrices $C$ in $\mathbb{S}_{3}^{+}$, we make use of the right $O(3)$-invariance of the Saint Venant-Kirchhoff density $W$ which is inherited by $Q W$. Therefore, $Q W(F)$ only depends on the singular values of $F$. We denote by $v_{1}(F) \leq v_{2}(F) \leq v_{3}(F)$ the singular values arranged in increasing order. It suffices to replace $c_{i i}$ by $v_{i}(F)^{2}$ in the above formulas to obtain an explicit expression for $Q W(F)$.

The expressions thus obtained are the same as those obtained in Le Dret and Raoult (1995a) that we recall below. First, for making comparisons 
between the energy and its quasiconvex envelop easier, we express the Saint Venant-Kirchhoff energy in terms of the singular values and we obtain

$$
W(F)=\frac{E}{8(1+\nu)} \sum_{i=1}^{3}\left(v_{i}(F)^{2}-1\right)^{2}+\frac{E \nu}{8(1+\nu)(1-2 \nu)}\left(\sum_{i=1}^{3} v_{i}(F)^{2}-3\right)^{2},
$$

where the Young modulus and the Poisson ratio are given by

$$
E=\frac{\mu(3 \lambda+2 \mu)}{\lambda+\mu} \text { and } \nu=\frac{\lambda}{2(\lambda+\mu)} .
$$

Let $\mathcal{T}=\left\{v \in \mathbb{R}^{3} ; 0 \leq v_{1} \leq v_{2} \leq v_{3}\right\}$ be the convex tetrahedral cone of $\mathbb{R}_{+}^{3}$ delimited by the planes $v_{1}=0, v_{1}=v_{2}$ and $v_{2}=v_{3}$. We define a mapping $q$ on $\mathcal{T}$ by

$$
\begin{gathered}
q(v)=\frac{E}{8}\left[v_{3}^{2}-1\right]_{+}^{2}+\frac{E}{8\left(1-\nu^{2}\right)}\left[v_{2}^{2}+\nu v_{3}^{2}-(1+\nu)\right]_{+}^{2} \\
+\frac{E}{8\left(1-\nu^{2}\right)(1-2 \nu)}\left[(1-\nu) v_{1}^{2}+\nu\left(v_{2}^{2}+v_{3}^{2}\right)-(1+\nu)\right]_{+}^{2},
\end{gathered}
$$

where $[x]_{+}^{2}=x^{2}$ if $x \geq 0,[x]_{+}^{2}=0$ if $x<0$. Previous computations allow to write the following theorem.

Theorem 1.7. The quasiconvex envelope of the Saint Venant-Kirchhoff stored energy function $W$ is given by

$$
\forall F \in \mathbb{M}_{3}, \quad Q W(F)=q\left(v_{1}(F), v_{2}(F), v_{3}(F)\right) .
$$

Let us examine more deeply the values taken by $Q W$. We introduce three subsets of $\mathcal{T}$ :

$$
\begin{gathered}
\mathcal{H}=\left\{v \in \mathcal{T} ; v_{3} \leq 1\right\}, \\
\mathcal{C}=\left\{v \in \mathcal{T} ; v_{2}^{2}+\nu v_{3}^{2}-(1+\nu) \leq 0\right\}, \\
\mathcal{E}=\left\{v \in \mathcal{T} ;(1-\nu) v_{1}^{2}+\nu\left(v_{2}^{2}+v_{3}^{2}\right)-(1+\nu) \leq 0\right\},
\end{gathered}
$$

which correspond to the various positive parts that appear in formula (16). It is easily checked that $\mathcal{H} \subset \mathcal{C} \subset \mathcal{E}$ and that

i) if $v \in \mathcal{H}$, then $q(v)=0$,

ii) if $v \in \mathcal{C} \backslash \mathcal{H}$, then $q(v)=\frac{E}{8}\left(v_{3}^{2}-1\right)^{2}$,

iii) if $v \in \mathcal{E} \backslash \mathcal{C}$, then

$$
q(v)=\frac{E}{8(1+\nu)} \sum_{i=2}^{3}\left(v_{i}^{2}-1\right)^{2}+\frac{E \nu}{8\left(1-\nu^{2}\right)}\left(\sum_{i=2}^{3} v_{i}^{2}-2\right)^{2},
$$


iv) if $v \notin \mathcal{E}$, then

$$
q(v)=\frac{E}{8(1+\nu)} \sum_{i=1}^{3}\left(v_{i}^{2}-1\right)^{2}+\frac{E \nu}{8(1+\nu)(1-2 \nu)}\left(\sum_{i=1}^{3} v_{i}^{2}-3\right)^{2} .
$$

For singular values outside of $\mathcal{E}$, the energy and its quasiconvex envelope coincide. At the other end of the scale, for sufficiently small singular values, the quasiconvex energy is equal to 0 . It is indeed a general fact that the quasiconvex envelope of a material indifferent and isotropic material vanishes on the set of singular values less than 1, see Le Dret and Raoult (1994).

\section{Quasiconvexity in the derivation of slender structure models}

In this section, we turn to the rigorous derivation of models for bidimensional structures from three-dimensional models. The idea of deriving simplified models from complete models goes back at least to the $50 \mathrm{~s}$ with the works by R.D. Mindlin and by E.Reissner among others. In the linear case, correct bidimensional models can be obtained by quick, but mathematically frightening ways: assuming for instance that the 33 component of the linearized strain tensor is equal to 0 in some equations of the three-dimensional model, but not in other ones. Later on, came the idea of considering a sequence of structures with thickness $2 \varepsilon$, of writing elasticity models for each of these structures and of studying the asymptotic behavior of the solutions of the models. Many researchers in applied mathematics worked on this subject and most of them consider that this procedure was first formalized by Ciarlet and Destuynder (1979).

The first result obtained by this method was of no surprise: the usual linear plate model is recovered. In the linear case, this line of work was pursued by identifying more precise models (i.e. not only identifying the limit of the three-dimensional solutions, but identifying a higher-order term as well), studying dynamical cases, or considering more general materials such that piezo-electric materials, visco-elastic materials. Things get trickier when dealing with nonlinear models: existence results are not always available and convergence proofs are much harder when products of terms have to be considered. Identifying limit models was first obtained by formal asymptotic methods on the system of partial differential equations describing finite elasticity written under variational form. Then came the realm of rigorous $\Gamma$-convergence arguments and their escort of quasiconvexification tools. The work we present here is taken from Le Dret and Raoult (1995a). 
We mention that a previous work by Acerbi et al. (1991) existed and, although in a one-dimesional setting that only requires convexity arguments, gave a path to follow.

\subsection{The three-dimensional and rescaled problems}

For all $\varepsilon>0$, let $\Omega_{\varepsilon}=\left\{x \in \mathbb{R}^{3} ;\left(x_{1}, x_{2}\right) \in \omega,\left|x_{3}\right|<\varepsilon\right\}$, where $\omega$ is an open, bounded subset of $\mathbb{R}^{2}$ with Lipschitz boundary. For all $z_{i} \in \mathbb{R}^{3}$, $i=1,2,3$, we note $\left(z_{1}\left|z_{2}\right| z_{3}\right)$ the $3 \times 3$ matrix whose $i$-th column is $z_{i}$. Let $W: \mathbb{M}_{3 \times 3} \mapsto \mathbb{R}$ be a continuous function that satisfies the following growth and coercivity hypotheses:

$$
\begin{gathered}
\exists C>0, \exists p \in] 1,+\infty\left[, \forall F \in \mathbb{M}_{3 \times 3},|W(F)| \leq C\left(1+\|F\|^{p}\right),\right. \\
\exists \alpha>0, \exists \beta \geq 0, \forall F \in \mathbb{M}_{3 \times 3}, W(F) \geq \alpha\|F\|^{p}-\beta .
\end{gathered}
$$

We assume that $\Omega_{\varepsilon}$ is the reference configuration of a hyperelastic homogeneous three-dimensional body whose stored energy function is $W$. We assume for simplicity that the bodies are submitted to the action of dead loading body force densities $f^{\varepsilon} \in L^{q}\left(\Omega_{\varepsilon} ; \mathbb{R}^{3}\right)$ and surface traction densities $g^{\varepsilon} \in L^{r}\left(S_{\varepsilon} ; \mathbb{R}^{3}\right)$ on $S_{\varepsilon}=\omega \times\{ \pm \varepsilon\}$, the top and bottom surfaces of $\Omega_{\varepsilon}$. For the sake of definiteness, we assume that $q=r$ and $1 / p+1 / q=1$, but other choices are indeed possible at no extra cost. Let $\left.\Gamma_{\varepsilon}=\partial \omega \times\right]-\varepsilon, \varepsilon[$ be the lateral surface of $\Omega_{\varepsilon}$. We assume that the deformations of the bodies satisfy a boundary condition of place on $\Gamma_{\varepsilon}$. The equilibrium problem may be formulated as a minimization problem:

$$
\text { Find } \phi^{\varepsilon} \in \Phi_{\varepsilon} \text { such that } I_{\varepsilon}\left(\phi^{\varepsilon}\right)=\inf _{\psi \in \Phi_{\varepsilon}} I_{\varepsilon}(\psi),
$$

where the total energy $I_{\varepsilon}$ is

$$
I_{\varepsilon}(\psi)=\int_{\Omega_{\varepsilon}} W(\nabla \psi) d x-\int_{\Omega_{\varepsilon}} f^{\varepsilon} \cdot \psi d x-\int_{S_{\varepsilon}} g^{\varepsilon} \cdot \psi d \sigma,
$$

and the set of admissible deformations is

$$
\Phi_{\varepsilon}=\left\{\psi \in W^{1, p}\left(\Omega_{\varepsilon} ; \mathbb{R}^{3}\right) ; \psi(x)=x \text { on } \Gamma_{\varepsilon}\right\} .
$$

We do not assume that $W$ is quasiconvex and problem (20) may well not possess any solutions. Naturally, if it does have solutions which are thus actual equilibrium deformations of the bodies, our results apply to these deformations.

Let us thus be given a diagonal minimizing sequence $\phi^{\varepsilon}$ for the sequence of energies $I_{\varepsilon}$ over the sets $\Phi_{\varepsilon}$. More specifically, we assume that

$$
\phi^{\varepsilon} \in \Phi_{\varepsilon}, \quad I_{\varepsilon}\left(\phi^{\varepsilon}\right) \leq \inf _{\psi \in \Phi_{\varepsilon}} I_{\varepsilon}(\psi)+\varepsilon s(\varepsilon),
$$


where $s$ is a positive function such that $s(\varepsilon) \rightarrow 0$ when $\varepsilon \rightarrow 0$. Such a sequence always exists and if the minimization problems have solutions, $\phi^{\varepsilon}$ may be chosen to be such a solution.

In order to obtain a membrane model in the limit, it is of crucial importance to specify the order of magnitude of the applied loads. In effect, it is always possible to stretch all thin cylinders $\Omega_{\varepsilon}$ into the same block, say $\Omega_{1}$, by applying sufficiently large forces. For such forces, the limit behavior is obviously not that of a membrane.

It turns out that the right order of magnitude is given by $\left\|f^{\varepsilon}\right\|_{L^{q}\left(\Omega_{\varepsilon} ; \mathbb{R}^{3}\right)} \leq$ $C \varepsilon^{1 / q}$ and $\left\|g^{\varepsilon}\right\|_{L^{q}\left(S_{\varepsilon} ; \mathbb{R}^{3}\right)} \leq C \varepsilon$ where the constant $C$ does not depend on $\varepsilon$. For example, the weight of the material, $f^{\varepsilon}(x)=(0,0,-\rho g)^{T}$, is allowed.

In order to rescale the problem, we let $\Omega=\Omega_{1}, \Gamma=\Gamma_{1}$ and $S=S_{1}$ and define a rescaling operator $\Theta_{\varepsilon}$ by $\left(\Theta_{\varepsilon} \psi\right)\left(x_{1}, x_{2}, x_{3}\right)=\psi\left(x_{1}, x_{2}, \varepsilon x_{3}\right)$. Let $\phi(\varepsilon)=\Theta_{\varepsilon} \phi^{\varepsilon}$ and $\phi_{0}(\varepsilon)(x)=\left(x_{1}, x_{2}, \varepsilon x_{3}\right)^{T}$. Note that all components are treated in the same way: we only transport $\phi^{\varepsilon}$ on the fixed domain $\Omega$. This is the same rescaling as that used in Fox et al. (1993). The rescaled displacement $u(\varepsilon)=\phi(\varepsilon)-\phi_{0}(\varepsilon)$ belongs to $V=W_{\Gamma}^{1, p}\left(\Omega ; \mathbb{R}^{3}\right)$. We accordingly rescale the energies by setting $I(\varepsilon)(\psi)=\varepsilon^{-1} I_{\varepsilon}\left(\Theta_{\varepsilon}^{-1} \psi\right)$, i.e.,

$$
I(\varepsilon)(\psi)=\int_{\Omega} W\left(\left(\partial_{1} \psi\left|\partial_{2} \psi\right| \frac{\partial_{3} \psi}{\varepsilon}\right)\right) d x-\int_{\Omega} f(\varepsilon) \cdot \psi d x-\int_{S} \varepsilon^{-1} g(\varepsilon) \cdot \psi d \sigma
$$

or in terms of the rescaled displacements

$$
\begin{array}{r}
J(\varepsilon)(v)=\int_{\Omega} W\left(\left(e_{1}+\partial_{1} v\left|e_{2}+\partial_{2} v\right| e_{3}+\frac{\partial_{3} v}{\varepsilon}\right)\right) d x \\
-\int_{\Omega} f(\varepsilon) \cdot\left(\phi_{0}(\varepsilon)+v\right) d x-\int_{S} \varepsilon^{-1} g(\varepsilon) \cdot\left(\phi_{0}(\varepsilon)+v\right) d \sigma
\end{array}
$$

where $f(\varepsilon)=\Theta_{\varepsilon} f^{\varepsilon}$ and $g(\varepsilon)=\Theta_{\varepsilon} g^{\varepsilon}$. It is immediate that

$$
J(\varepsilon)(u(\varepsilon)) \leq \inf _{v \in V} J(\varepsilon)(v)+s(\varepsilon)
$$

For simplicity, we assume that $f(\varepsilon)=f$ and $\varepsilon^{-1} g(\varepsilon)=g$ are independent of $\varepsilon$.

\subsection{Computation of the $\Gamma$-limit of the rescaled energies}

We use $\Gamma$-convergence theory to determine the asymptotic behavior of the rescaled displacements $u(\varepsilon)$ when $\varepsilon \rightarrow 0$. In the sequel, the thickness parameter $\varepsilon$ will take its values in a sequence $\varepsilon_{n} \rightarrow 0$. Since the results 
do not depend on the sequence in question, and for notational brevity, we will simply use the notation $\varepsilon$. Let us recall that a sequence of functions $G_{\varepsilon}$ from a metric space $X$ into $\overline{\mathbb{R}}$ is said to $\Gamma$-converge toward $G_{0}$ for the topology of $X$ if the following two conditions are satisfied for all $x \in X$ :

$$
\begin{gathered}
\forall x_{\varepsilon} \rightarrow x, \liminf G_{\varepsilon}\left(x_{\varepsilon}\right) \geq G_{0}(x), \\
\exists y_{\varepsilon} \rightarrow x, G_{\varepsilon}\left(y_{\varepsilon}\right) \rightarrow G_{0}(x) .
\end{gathered}
$$

If the sequence $G_{\varepsilon} \Gamma$-converges, its $\Gamma$-limit is lower semicontinuous and is alternatively given by

$$
G_{0}(x)=\min \left\{\liminf G_{\varepsilon}\left(x_{\varepsilon}\right) ; x_{\varepsilon} \rightarrow x\right\} .
$$

In addition, the set of functions from $X$ into $\overline{\mathbb{R}}$ has a sequential compactness property with respect to $\Gamma$-convergence in the sense that any sequence $G_{\varepsilon}: X \rightarrow \overline{\mathbb{R}}$ admits a $\Gamma$-convergent subsequence. The main interest of $\Gamma$-convergence is that if the minimizers of $G_{\varepsilon}$ stay in a compact set of $X$ for all $\varepsilon$, then their limit points are minimizers of $G_{0}$, see De Giorgi and Franzoni (1975), Attouch (1984), Dal Maso (1993).

We do not use $J(\varepsilon)$ directly, since this would imply working with the weak topology of $W^{1, p}\left(\Omega ; \mathbb{R}^{3}\right)$, which is non metrizable. Instead, we extend the energies to $L^{p}\left(\Omega ; \mathbb{R}^{3}\right)$ by setting

$$
\forall v \in L^{p}\left(\Omega ; \mathbb{R}^{3}\right), \tilde{J}(\varepsilon)(v)=J(\varepsilon)(v) \text { if } v \in V, \quad+\infty \text { otherwise. }
$$

This is a classical trick used in the applications of $\Gamma$-convergence: obviously, this does not change the minimization problem. It has the additional virtue of incorporating the boundary conditions in the energy functional.

Let us now proceed to compute the $\Gamma$-limit of the sequence $\tilde{J}(\varepsilon)$ for the strong topology of $L^{p}\left(\Omega ; \mathbb{R}^{3}\right)$. Let $\mathbb{M}_{3 \times 2}$ be the space of $3 \times 2$ real matrices endowed with the usual Euclidean norm $\|\bar{F}\|=\sqrt{\operatorname{tr}\left(\bar{F}^{T} \bar{F}\right)}$. We note $\left(z_{1} \mid z_{2}\right)$ the matrix of $\mathbb{M}_{3 \times 2}$ whose $\alpha$-th column is $z_{\alpha} \in \mathbb{R}^{3}$. For all $\bar{F}=\left(z_{1} \mid z_{2}\right) \in \mathbb{M}_{3 \times 2}$ and $z \in \mathbb{R}^{3}$, we also note $(\bar{F} \mid z)$ the matrix whose first two columns are $z_{1}$ and $z_{2}$ and whose third column is $z$.

As in Acerbi et al. (1991) for elastic strings, we define $W_{0}: \mathbb{M}_{3 \times 2} \rightarrow \mathbb{R}$ by

$$
W_{0}(\bar{F})=\inf _{z \in \mathbb{R}^{3}} W((\bar{F} \mid z)) .
$$

Due to the coercivity assumption on $W$, it is clear that this function is well defined. Besides, since $W$ is continuous, the infimum is attained. 
Proposition 2.1. The function $W_{0}$ is continuous and satisfies the growth and coercivity estimates:

$$
\begin{gathered}
\exists C^{\prime}>0, \forall \bar{F} \in \mathbb{M}_{3 \times 2},\left|W_{0}(\bar{F})\right| \leq C^{\prime}\left(1+\|\bar{F}\|^{p}\right), \\
\forall \bar{F} \in \mathbb{M}_{3 \times 2}, W_{0}(\bar{F}) \geq \alpha\|\bar{F}\|^{p}-\beta .
\end{gathered}
$$

Proof - Since $W_{0}$ is an infimum of continuous functions, it is upper semicontinuous. Let $\bar{F} \in \mathbb{M}_{3 \times 2}$ and consider a sequence $\bar{F}^{n} \in \mathbb{M}_{3 \times 2}$ such that $\bar{F}^{n} \rightarrow \bar{F}$ as $n \rightarrow+\infty$. Because of the coercivity assumption on $W$, there exists a compact set $K$ such that for all $\bar{F}^{n}$ the infimum in definition (27) is attained at a point $z^{n} \in K$. Consider a subsequence, still denoted $n$, such that $W_{0}\left(\bar{F}^{n}\right)$ converges when $n \rightarrow+\infty$. We extract a further subsequence such that $z^{n} \rightarrow z \in K$. By continuity of $W, W_{0}\left(\bar{F}^{n}\right)=$ $W\left(\left(\bar{F}^{n} \mid z^{n}\right)\right) \rightarrow W((\bar{F} \mid z)) \geq W_{0}(\bar{F})$. As this is true for all subsequences such that $W_{0}\left(\bar{F}^{n}\right)$ converges, it follows that liminf $W_{0}\left(\bar{F}^{n}\right) \geq W_{0}(\bar{F})$, hence $W_{0}$ is lower semicontinuous.

For all $\bar{F} \in \mathbb{M}_{3 \times 2}$, let $z_{0}$ be a point where the infimum in definition (27) is attained. Thus, $W_{0}(\bar{F})=W\left(\left(\bar{F} \mid z_{0}\right)\right) \geq \alpha\left\|\left(\bar{F} \mid z_{0}\right)\right\|^{p}-\beta \geq \alpha\|\bar{F}\|^{p}-\beta$. Hence $W_{0}$ is coercive. Therefore, $W_{0}$ is nonnegative outside of a compact set $K^{\prime}$. Since $\left|W_{0}\right|$ is continuous, it is bounded on $K^{\prime}$ and for $\bar{F} \notin K^{\prime}$, $\left|W_{0}(\bar{F})\right|=W_{0}(\bar{F}) \leq W((\bar{F} \mid 0)) \leq C\left(1+\|(\bar{F} \mid 0)\|^{p}\right)=C\left(1+\|\bar{F}\|^{p}\right)$, which proves the growth estimate.

Let $Q W_{0}=\sup \left\{Z: \mathbb{M}_{3 \times 2} \rightarrow \mathbb{R}, Z\right.$ quasiconvex, $\left.Z \leq W_{0}\right\}$ be the quasiconvex envelope of $W_{0}$. Let us introduce the space

$$
V_{M}=\left\{v \in V ; \partial_{3} v=0\right\},
$$

which we call the space of membrane displacements. It is canonically isomorphic to $W_{0}^{1, p}\left(\omega ; \mathbb{R}^{3}\right)$ and we let $\bar{v}$ denote the element of $W_{0}^{1, p}\left(\omega ; \mathbb{R}^{3}\right)$ that is associated with $v \in V_{M}$ through this isomorphism. The expression of the $\Gamma$-limit of the sequence $\tilde{J}(\varepsilon)$ is given in the following theorem.

Theorem 2.2. The sequence $\tilde{J}(\varepsilon) \Gamma$-converges for the strong topology of $L^{p}\left(\Omega ; \mathbb{R}^{3}\right)$ when $\varepsilon \rightarrow 0$. Let $\tilde{J}(0)$ be its $\Gamma$-limit. For all $v \in L^{p}\left(\Omega ; \mathbb{R}^{3}\right) \cap V_{M}$,

$$
\tilde{J}(0)(v)=2 \int_{\omega} Q W_{0}\left(\left(e_{1}+\partial_{1} \bar{v} \mid e_{2}+\partial_{2} \bar{v}\right)\right) d x_{1} d x_{2}-\int_{\omega} \mathcal{F} \cdot\left(\phi_{0}(0)+\bar{v}\right) d x_{1} d x_{2}
$$

where $\mathcal{F}\left(x_{1}, x_{2}\right)=\int_{-1}^{1} f\left(x_{1}, x_{2}, x_{3}\right) d x_{3}+g\left(x_{1}, x_{2}, 1\right)+g\left(x_{1}, x_{2},-1\right)$, and $\tilde{J}(0)(v)=+\infty$ if $v \in L^{p}\left(\Omega ; \mathbb{R}^{3}\right) \backslash V_{M}$. 
For clarity, we break the proof of Theorem 2.2 into a series of lemmas and propositions.

We begin by extracting a $\Gamma$-convergent subsequence and call $\tilde{J}(0)$ its $\Gamma$ limit. The uniqueness of $\widetilde{J}(0)$ will make the extraction of this subsequence superfluous a posteriori.

Lemma 2.3. Let $v(\varepsilon) \in L^{p}\left(\Omega ; \mathbb{R}^{3}\right)$ be a sequence such that $\tilde{J}(\varepsilon)(v(\varepsilon)) \leq$ $C<+\infty$ where $C$ does not depend on $\varepsilon$. Then $v(\varepsilon)$ is uniformly bounded in $V$ and its limit points for the weak topology of $V$ belong to $V_{M}$.

Proof- Let $v(\varepsilon) \in L^{p}\left(\Omega ; \mathbb{R}^{3}\right)$ be such that $\tilde{J}(\varepsilon)(v(\varepsilon)) \leq C<+\infty$. Then, the definition (26) of the function $\tilde{J}(\varepsilon)$ implies first of all that $v(\varepsilon) \in V$ for all $\varepsilon>0$. Let us call $\psi(\varepsilon)=v(\varepsilon)+\phi_{0}(\varepsilon)$ the deformation that is associated with the displacement $v(\varepsilon)$. The coercivity of the function $W$ and the assumed uniform bound for the energies imply that

$$
\alpha \int_{\Omega}\left\|\left(\partial_{1} \psi(\varepsilon)\left|\partial_{2} \psi(\varepsilon)\right| \varepsilon^{-1} \partial_{3} \psi(\varepsilon)\right)\right\|^{p} d x \leq C^{\prime}\left(1+\|\psi(\varepsilon)\|_{W^{1, p}\left(\Omega ; \mathbb{R}^{3}\right)}\right)
$$

where $C^{\prime}$ does not depend on $\varepsilon$. It is clear that for all $\varepsilon \leq 1,\left\|\left(z_{1}\left|z_{2}\right| \varepsilon^{-1} z_{3}\right)\right\| \geq$ $\left\|\left(z_{1}\left|z_{2}\right| z_{3}\right)\right\|$. Therefore, (32) implies that

$$
\alpha\|\nabla \psi(\varepsilon)\|_{L^{p}\left(\Omega ; \mathbb{R}^{3}\right)}^{p} \leq C^{\prime}\left(1+\|\psi(\varepsilon)\|_{W^{1, p}\left(\Omega ; \mathbb{R}^{3}\right)}\right),
$$

which, together with the boundary condition of place $\psi(\varepsilon)=\phi_{0}(\varepsilon)$ on $\Gamma$, yields the desired uniform bound for $\psi(\varepsilon)$ in $W^{1, p}\left(\Omega ; \mathbb{R}^{3}\right)$ by Poincaré's inequality. Since $\phi_{0}(\varepsilon)$ is obviously uniformly bounded in $W^{1, p}\left(\Omega ; \mathbb{R}^{3}\right)$, the same holds true for $v(\varepsilon)$.

On the other hand, since $\left\|\left(z_{1}\left|z_{2}\right| \varepsilon^{-1} z_{3}\right)\right\| \geq \varepsilon^{-1}\left|z_{3}\right|$, where $|\cdot|$ denotes the Euclidean norm into $\mathbb{R}^{3}$, upon using the bound just established above in inequality (33) we obtain that $\left\|\partial_{3} \psi(\varepsilon)\right\|_{L^{p}\left(\Omega ; \mathbb{R}^{3}\right)} \leq C^{\prime \prime} \varepsilon$, so that $\partial_{3} \psi(\varepsilon) \rightarrow 0$ strongly in $L^{p}\left(\Omega ; \mathbb{R}^{3}\right)$. If we let $\psi$ denote any limit point of the sequence $\psi(\varepsilon)$ for the weak topology of $W^{1, p}\left(\Omega ; \mathbb{R}^{3}\right)$, it follows at once that $\partial_{3} \psi=0$. If $v$ denotes the corresponding limit point of the sequence $v(\varepsilon)$, since $v=$ $\psi-\phi_{0}(0)$ and $\partial_{3} \phi_{0}(0)=0$, we obtain that $v$ belongs to $V_{M}$.

Corollary 2.4. If $v \in L^{p}\left(\Omega ; \mathbb{R}^{3}\right)$ but $v \notin V_{M}$, then $\tilde{J}(0)(v)=+\infty$.

Proof - Indeed, if $\tilde{J}(0)(v)<+\infty$, there exists a sequence $v(\varepsilon)$ that converges strongly to $v$ in $L^{p}\left(\Omega ; \mathbb{R}^{3}\right)$ and such that $\tilde{J}(\varepsilon)(v(\varepsilon)) \rightarrow \tilde{J}(0)(v)$. Therefore, by Lemma 2.3, $v \in V_{M}$.

We thus only have to compute the value of the $\Gamma$-limit for displacements in $V_{M}$. 
Proposition 2.5. For all $v \in V_{M}$, we have that

$$
\tilde{J}(0)(v) \geq 2 \int_{\omega} Q W_{0}\left(\left(e_{1}+\partial_{1} \bar{v} \mid e_{2}+\partial_{2} \bar{v}\right)\right) d x_{1} d x_{2}-\int_{\omega} \mathcal{F} \cdot\left(\phi_{0}(0)+\bar{v}\right) d x_{1} d x_{2} .
$$

Proof - Consider any $v \in V_{M}$. Since $J(\varepsilon)(v)$ is obviously bounded from above independently of $\varepsilon$, it follows that $\tilde{J}(0)(v)<+\infty$. By the definition of $\Gamma$-convergence, there exists a sequence $v(\varepsilon) \in V$ such that $v(\varepsilon) \rightarrow v$ strongly in $L^{p}\left(\Omega ; \mathbb{R}^{3}\right)$ and $\tilde{J}(\varepsilon)(v(\varepsilon)) \rightarrow \tilde{J}(0)(v)$. Then, by Lemma 2.3, $v(\varepsilon) \rightarrow v$ weakly in $V$.

First of all, it is clear that, when $\varepsilon \rightarrow 0$,

$$
\int_{\Omega} f \cdot\left(\phi_{0}(\varepsilon)+v(\varepsilon)\right) d x+\int_{S} g \cdot\left(\phi_{0}(\varepsilon)+v(\varepsilon)\right) d \sigma \rightarrow \int_{\omega} \mathcal{F} \cdot\left(\phi_{0}(0)+\bar{v}\right) d x_{1} d x_{2} .
$$

For the elastic energy, we have that (with $\psi(\varepsilon)=v(\varepsilon)+\phi_{0}(\varepsilon)$ as usual)

$$
\begin{aligned}
\int_{\Omega} W\left(\left(\partial_{1} \psi(\varepsilon)\left|\partial_{2} \psi(\varepsilon)\right| \frac{\partial_{3} \psi(\varepsilon)}{\varepsilon}\right)\right) d x & \geq \int_{\Omega} W_{0}\left(\left(\partial_{1} \psi(\varepsilon) \mid \partial_{2} \psi(\varepsilon)\right)\right) d x \\
& \geq \int_{\Omega} Q W_{0}\left(\left(\partial_{1} \psi(\varepsilon) \mid \partial_{2} \psi(\varepsilon)\right)\right) d x
\end{aligned}
$$

Let $G: W^{1, p}\left(\Omega ; \mathbb{R}^{3}\right) \mapsto \mathbb{R}$ be defined by

$$
G(\psi)=\int_{\Omega} Q W_{0}\left(\left(\partial_{1} \psi \mid \partial_{2} \psi\right)\right) d x
$$

Let us define a function $Z: \mathbb{M}_{3} \mapsto \mathbb{R}$ by $Z\left(\left(z_{1}\left|z_{2}\right| z_{3}\right)\right)=Q W_{0}\left(\left(z_{1} \mid z_{2}\right)\right)$. Since $Q W_{0}$ is quasiconvex, $Z$ is also quasiconvex. Indeed, let $d$ be the unit square in $\mathbb{R}^{2}$ and $\left.D=d \times\right] 0,1\left[\right.$. Consider any function $\phi \in \mathcal{D}\left(D ; \mathbb{R}^{3}\right)$. For all $y \in] 0,1\left[\right.$, the function $\phi_{y}$ defined by $\phi_{y}\left(x_{1}, x_{2}\right)=\phi\left(x_{1}, x_{2}, y\right)$ belongs to $\mathcal{D}\left(d ; \mathbb{R}^{3}\right)$. Hence, for all $F \in \mathbb{M}_{3}$,

$$
\begin{aligned}
\int_{D} Z(F+\nabla \phi) d x & =\int_{D} Q W_{0}\left(\left(z_{1}+\partial_{1} \phi \mid z_{2}+\partial_{2} \phi\right)\right) d x \\
& =\int_{0}^{1}\left(\int_{d} Q W_{0}\left(\left(z_{1}+\partial_{1} \phi_{y} \mid z_{2}+\partial_{2} \phi_{y}\right)\right) d x_{1} d x_{2}\right) d y \\
& \geq \int_{0}^{1} Q W_{0}\left(\left(z_{1} \mid z_{2}\right)\right) d y=Z(F) .
\end{aligned}
$$

This implies that $Z$ is quasiconvex.

We now remark that $Z$ is quasiconvex, bounded below by $-\beta$ and satisfies the growth condition (18) since $Q W_{0}$ satisfies (28). Therefore, the 
function $G$ is sequentially weakly lower semicontinuous on $W^{1, p}\left(\Omega ; \mathbb{R}^{3}\right)$, Acerbi and Fusco (1984). Consequently, since $\psi(\varepsilon) \rightarrow \psi=v+\phi_{0}(0)$ in $W^{1, p}\left(\Omega ; \mathbb{R}^{3}\right)$,

$$
\begin{aligned}
& \liminf _{\varepsilon \rightarrow 0} \int_{\Omega} W\left(\left(\partial_{1} \psi(\varepsilon)\left|\partial_{2} \psi(\varepsilon)\right| \frac{\partial_{3} \psi(\varepsilon)}{\varepsilon}\right)\right) d x \geq \liminf _{\varepsilon \rightarrow 0} G(\psi(\varepsilon)) \\
& \geq G(\psi)=2 \int_{\omega} Q W_{0}\left(\left(e_{1}+\partial_{1} \bar{v} \mid e_{2}+\partial_{2} \bar{v}\right)\right) d x
\end{aligned}
$$

and the proof is complete.

Let us now turn to proving the reverse inequality.

Proposition 2.6. For all $v \in V_{M}$, we have that

$$
\tilde{J}(0)(v) \leq 2 \int_{\omega} Q W_{0}\left(\left(e_{1}+\partial_{1} \bar{v} \mid e_{2}+\partial_{2} \bar{v}\right)\right) d x_{1} d x_{2}-\int_{\omega} \mathcal{F} \cdot\left(\phi_{0}(0)+\bar{v}\right) d x_{1} d x_{2} .
$$

Proof - Let us consider $v \in V_{M}$. For all $w \in W_{0}^{1, p}\left(\omega ; \mathbb{R}^{3}\right)$, we define a displacement

$$
v(\varepsilon)(x)=\bar{v}\left(x_{1}, x_{2}\right)+\varepsilon x_{3} w(x) .
$$

Obviously, $v(\varepsilon) \rightarrow v$ strongly in $W^{1, p}\left(\Omega ; \mathbb{R}^{3}\right)$. Let us examine the limit behavior of the sequence $\tilde{J}(\varepsilon)(v(\varepsilon))$. By the dominated convergence theorem and the growth estimate, it is clear that

$$
\int_{\Omega} W\left(\left(\partial_{1}\left(\bar{\psi}+\varepsilon x_{3} w\right)\left|\partial_{2}\left(\bar{\psi}+\varepsilon x_{3} w\right)\right| e_{3}+w\right)\right) d x \rightarrow \int_{\Omega} W\left(\left(\partial_{1} \bar{\psi}\left|\partial_{2} \bar{\psi}\right| e_{3}+w\right)\right) d x
$$

when $\varepsilon \rightarrow 0$. Consequently,

$$
\tilde{J}(\varepsilon)(v(\varepsilon)) \rightarrow \int_{\Omega} W\left(\left(\partial_{1} \bar{\psi}\left|\partial_{2} \bar{\psi}\right| e_{3}+w\right)\right) d x-\int_{\omega} \mathcal{F} \cdot\left(\phi_{0}(0)+\bar{v}\right) d x_{1} d x_{2} .
$$

As this is true for all $w \in W_{0}^{1, p}\left(\omega ; \mathbb{R}^{3}\right)$, it follows from the definition of $\Gamma$-convergence that

$$
\tilde{J}(0)(v) \leq \inf _{w \in W_{0}^{1, p}\left(\omega ; \mathbb{R}^{3}\right)} \int_{\Omega} W\left(\left(\partial_{1} \bar{\psi}\left|\partial_{2} \bar{\psi}\right| e_{3}+w\right)\right) d x-\int_{\omega} \mathcal{F} \cdot\left(\phi_{0}(0)+\bar{v}\right) d x_{1} d x_{2} .
$$

We remark that

$$
\inf _{W_{0}^{1, p}\left(\omega ; \mathbb{R}^{3}\right)} \int_{\Omega} W\left(\left(\partial_{1} \bar{\psi}\left|\partial_{2} \bar{\psi}\right| e_{3}+w\right)\right) d x=\inf _{L^{p}\left(\omega ; \mathbb{R}^{3}\right)} \int_{\Omega} W\left(\left(\partial_{1} \bar{\psi}\left|\partial_{2} \bar{\psi}\right| e_{3}+w\right)\right) d x,
$$


by the density of $W_{0}^{1, p}\left(\omega ; \mathbb{R}^{3}\right)$ in $L^{p}\left(\omega ; \mathbb{R}^{3}\right)$ and by the dominated convergence theorem. The function $g: \omega \times \mathbb{R}^{3} \rightarrow \mathbb{R}$ defined by $g(x, z)=$ $W\left(\left(\partial_{1} \bar{\psi}(x)\left|\partial_{2} \bar{\psi}(x)\right| e_{3}+z\right)\right)$ is a Carathéodory function. Hence, the measurable selection lemma, Ekeland and Temam (1974), shows that there exists a measurable function $w_{0}$ such that

$$
W_{0}\left(\left(\partial_{1} \bar{\psi}(x) \mid \partial_{2} \bar{\psi}(x)\right)\right)=W\left(\left(\partial_{1} \bar{\psi}(x)\left|\partial_{2} \bar{\psi}(x)\right| e_{3}+w_{0}(x)\right)\right)
$$

for almost all $x \in \omega$. Due to the coercivity estimate, $w_{0} \in L^{p}\left(\omega ; \mathbb{R}^{3}\right)$ and thus

$$
\inf _{w \in L^{p}\left(\omega ; \mathbb{R}^{3}\right)} \int_{\Omega} W\left(\left(\partial_{1} \bar{\psi}\left|\partial_{2} \bar{\psi}\right| e_{3}+w\right)\right) d x \leq \int_{\Omega} W_{0}\left(\left(\partial_{1} \bar{\psi} \mid \partial_{2} \bar{\psi}\right)\right) d x .
$$

Let $G: W_{0}^{1, p}\left(\omega ; \mathbb{R}^{3}\right) \rightarrow \mathbb{R}$ be defined by

$$
G(\bar{v})=2 \int_{\omega} W_{0}\left(\left(\partial_{1} \bar{\psi} \mid \partial_{2} \bar{\psi}\right)\right) d x_{1} d x_{2}-\int_{\omega} \mathcal{F} \cdot\left(\phi_{0}(0)+\bar{v}\right) d x_{1} d x_{2} .
$$

It follows from (40) that for all $v \in V_{M}$

$$
\tilde{J}(0)(v) \leq G(\bar{v}) .
$$

Since $\tilde{J}(0)$ is lower semicontinuous on $L^{p}\left(\Omega ; \mathbb{R}^{3}\right)$, its restriction to $V_{M}$ is sequentially weakly lower semicontinuous on $V_{M}$ equipped with the $W^{1, p}\left(\omega ; \mathbb{R}^{3}\right)$ topology. Therefore,

$$
\tilde{J}(0)_{\left.\right|_{V_{M}}} \leq \Gamma-G
$$

where $\Gamma-G$ is the sequential weak lower semicontinuous envelope of $G$ on $W_{0}^{1, p}\left(\omega ; \mathbb{R}^{3}\right)$. It is known, see Acerbi and Fusco (1984), that $\Gamma-G$ is given by

$$
\Gamma-G(\bar{v})=2 \int_{\omega} Q W_{0}\left(\left(\partial_{1} \bar{\psi} \mid \partial_{2} \bar{\psi}\right)\right) d x_{1} d x_{2}-\int_{\omega} \mathcal{F} \cdot\left(\phi_{0}(0)+\bar{v}\right) d x_{1} d x_{2} .
$$

which proves proposition 2.6 .

Theorem 2.2 is proved by gathering all previous results.

\subsection{Convergence of the rescaled deformations and the nonlinear membrane model}

We now use Theorem 2.2 to characterize the asymptotic behavior of diagonal minimizing sequences of rescaled deformations $\phi(\varepsilon)$ for the sequence of rescaled energies $I(\varepsilon)$, which are such that $I(\varepsilon)(\phi(\varepsilon)) \leq \inf _{\psi \in \Phi(\varepsilon)} I(\varepsilon)(\psi)+h(\varepsilon)$ 
where $h$ is a positive function such that $h(\varepsilon) \rightarrow 0$ when $\varepsilon \rightarrow 0$ and the sets of admissible deformations are

$$
\Phi(\varepsilon)=\left\{\psi \in W^{1, p}\left(\Omega ; \mathbb{R}^{3}\right) ; \psi(x)=\phi_{0}(\varepsilon)(x) \text { on } \Gamma\right\} .
$$

We introduce the space of membrane deformations as $\Phi_{M}=\{\psi \in$ $W^{1, p}\left(\Omega ; \mathbb{R}^{3}\right), \partial_{3} \psi=0, \psi(x)=\left(x_{1}, x_{2}, 0\right)^{T}$ on $\left.\Gamma\right\}$, which is isomorphic to $\bar{\Phi}_{M}=\left\{\bar{\psi} \in W^{1, p}\left(\omega ; \mathbb{R}^{3}\right), \bar{\psi}\left(x_{1}, x_{2}\right)=\left(x_{1}, x_{2}, 0\right)^{T}\right.$ on $\left.\partial \omega\right\}$.

Theorem 2.7. The sequence $\phi(\varepsilon)$ is relatively weakly compact in $W^{1, p}\left(\Omega ; \mathbb{R}^{3}\right)$. Its limit points $\phi$ belong to $\Phi_{M}$ and are identified with elements $\bar{\phi}$ of $\bar{\Phi}_{M}$, solutions of the minimization problem $\bar{I}(0)(\bar{\phi})=\inf _{\bar{\psi} \in \bar{\Phi}_{M}} \bar{I}(0)(\bar{\psi})$, where the membrane energy $\bar{I}(0)$ is given by

$$
\bar{I}(0)(\bar{\psi})=2 \int_{\omega} Q W_{0}(\nabla \bar{\psi}) d x_{1} d x_{2}-\int_{\omega} \mathcal{F} \cdot \bar{\psi} d x_{1} d x_{2} .
$$

Proof - We follow an argument due to De Giorgi. With the rescaled deformation $\phi(\varepsilon)$, we associate a rescaled displacement $u(\varepsilon) \in V$ by $u(\varepsilon)=$ $\phi(\varepsilon)-\phi_{0}(\varepsilon)$. Obviously, $J(\varepsilon)(u(\varepsilon)) \leq \inf _{v \in V} J(\varepsilon)(v)+h(\varepsilon)$. In particular, $J(\varepsilon)(u(\varepsilon))$ is uniformly bounded and Lemma 2.3 shows that the sequence $u(\varepsilon)$ is relatively weakly compact in $V$ and that its limit points belong to $V_{M}$. This implies in particular that $u(\varepsilon)$ is relatively compact in $L^{p}\left(\Omega ; \mathbb{R}^{3}\right)$.

On the other hand, it is clear that $\tilde{J}(\varepsilon)(u(\varepsilon)) \leq \inf _{v \in L^{p}\left(\Omega ; \mathbb{R}^{3}\right)} \tilde{J}(\varepsilon)(v)+$ $h(\varepsilon)$. Let $u$ be a limit point of $u(\varepsilon)$ for the strong topology of $L^{p}\left(\Omega ; \mathbb{R}^{3}\right)$ (such a $u$ is also a limit point of $u(\varepsilon)$ for the weak topology of $V)$. Without loss of generality, we may assume that $u(\varepsilon) \rightarrow u$ strongly in $L^{p}\left(\Omega ; \mathbb{R}^{3}\right)$. Let $v$ be an arbitrary element of $L^{p}\left(\Omega ; \mathbb{R}^{3}\right)$ and consider a sequence $v(\varepsilon) \in L^{p}\left(\Omega ; \mathbb{R}^{3}\right)$ such that

$$
v(\varepsilon) \rightarrow v, \tilde{J}(\varepsilon)(v(\varepsilon)) \rightarrow \tilde{J}(0)(v) .
$$

Such a sequence exists by the very definition of $\Gamma$-convergence. Since $\tilde{J}(\varepsilon)(u(\varepsilon)) \leq \tilde{J}(\varepsilon)(v(\varepsilon))+h(\varepsilon)$, it follows that

$$
\tilde{J}(0)(u) \leq \liminf \tilde{J}(\varepsilon)(u(\varepsilon)) \leq \liminf (\tilde{J}(\varepsilon)(v(\varepsilon))+h(\varepsilon))=\tilde{J}(0)(v) .
$$

Therefore, $u$ is a minimizer of $\tilde{J}(0)$. Rewriting this statement in terms of $\bar{\phi}$, we obtain Theorem 2.7.

Remark 2.8. i) It is classical in $\Gamma$-convergence theory that the energies converge as well, in the sense that $I(\varepsilon)(\phi(\varepsilon)) \rightarrow \bar{I}(0)(\bar{\phi})$.

ii) If the three-dimensional elasticity problem has solutions that minimize the elastic energy, Theorem 2.7 applies to these minimizers. Such is 
the case for the polyconvex stored energy functions. Examples of realistic stored energy functions that satisfy the above hypotheses include Ogden's stored energy functions with appropriate exponents. For a derivation of the membrane model under the constraint $\operatorname{det}(\nabla \phi) \neq 0$, see Anza Hafsa and Mandallena (2006).

iii) The weak limits $\phi$ of weakly convergent subsequences of $\phi(\varepsilon)$ do not depend on $x_{3}$, in which sense the problem becomes two-dimensional in the limit. If regular enough, the associated function $\bar{\phi}$ describes a deformation of $\omega$ into a surface of $\mathbb{R}^{3}$. The elastic energy of such a deformation, $2 \int_{\omega} Q W_{0}(\nabla \bar{\phi}) d x_{1} d x_{2}$, only depends on its first derivatives. There are thus no bending effects associated with curvature, in which sense the resulting model is a nonlinear membrane model.

iv) If the function $Q W_{0}$ is smooth enough, the Euler-Lagrange equations for the membrane problem assume the form

$$
-\partial_{\beta}\left(\frac{\partial}{\partial \bar{F}} Q W_{0}(\nabla \bar{\phi})\right)_{i \beta}=\frac{1}{2} \mathcal{F}_{i} \text { in } \omega, \quad \bar{\phi}\left(x_{1}, x_{2}\right)=\left(x_{1}, x_{2}, 0\right)^{T} \text { on } \partial \omega,
$$

where Greek indices take their values in the set $\{1,2\}$, Latin indices in the set $\{1,2,3\}$ and the summation convention is understood. System (43) is a system of three quasilinear partial differential equations in the three unknowns $\bar{\phi}_{i}$. If the applied load is the weight, assuming the membrane is horizontal in its reference configuration, the limit load is of the form $1 / 2 \mathcal{F}=(0,0,-\rho g)^{T}$. The function $\bar{F} \mapsto \bar{T}_{R}(\bar{F})=\frac{\partial}{\partial \bar{F}} Q W_{0}(\bar{F})$ appears as the constitutive law for the analogue of the first Piola-Kirchoff stress tensor in the membrane. It gives the Lagrangian description of the tensile stresses in the membrane. It appears that the limit membrane problem retains the full quasilinear structure of three-dimensional elasticity, in contrast with such nonlinear plate models as the von Kármán equations that are only semilinear.

v) The explicit computation of $Q W_{0}$ when $W$ is the three-dimensional Saint Venant Kirchhoff energy is given in Le Dret and Raoult (1995a).

vi) The formal derivation of two-dimensional bending models was obtained in Fox et al. (1993) by asymptotic expansions. The bending model was there shown to be part of a hierarchy of models starting with the membrane model and ending with the linear plate model. Preliminary results for a rigorous derivation of the bending model by $\Gamma$-convergence arguments were given in Pantz (2001). The issue was completely settled in Friesecke et al. (2002) where an important rigidity lemma is proved. See also Friesecke et al. (2006).

vii) The previous method can be adapted to the study of the consistency of director models (Cosserat theory). In such models, the deformation is 
assumed to have a specific form. Namely, the deformation of each point $\left(x_{1}, x_{2}, x_{3}\right)$ of the three-dimensional body is assumed to be the sum of the deformation of point $\left(x_{1}, x_{2}\right)$ and of a term colinear to a vector depending on $\left(x_{1}, x_{2}\right)$ only, called the director vector. Some models assume the director to be of norm 1, or to remain normal to the deformed configuration. We proved in Le Dret and Raoult (2000) that the inextensibility constraint is inadequate in the membrane regime. On the contrary, the unconstrained Cosserat hypothesis provides a correct framework to account for the nonlinear membrane behavior. In order to obtain information on the asymptotic behavior of the director field, we had to study the weak lower semicontinuity of integral functionals of the form

$$
I(\psi, \delta)=\int_{\omega} W(\nabla \psi, \delta) d x
$$

where $\psi: \omega \subset \mathbb{R}^{2} \mapsto \mathbb{R}^{3}, \delta: \omega \mapsto \mathbb{R}^{3}$. This led us to introduce the notion of cross-quasiconvexity which is related to $A$-convexity, see Fonseca and Müller (1999).

\section{Quasiconvexity in the derivation of lattice homogenized models}

This section deals with lattices. For the sake of definiteness, we study bidimensional lattices that can deform into $\mathbb{R}^{3}$. Given a lattice made of bars and nodes, or given an atom lattice, we aim at deriving a continuous model whose macroscopic behavior is similar to the discrete lattice behavior. The discrete model we start from takes into account the changes of lengths as well as the changes of angles under deformation. Formal results in this setting were first given in Caillerie et al. (2003) and in Mourad (2003) and they are valid for any lattice. They were detailed later on in Caillerie et al. (2006) for graphene sheets whose carbon atoms make hexagonal networks. The rigorous results we present here are valid for a restricted class of lattices, square lattices are a good example of. An extended version will be published in a joint work with Nicolas Meunier and Olivier Pantz.

\subsection{The lattice energy}

We consider a discrete two-dimensional lattice. Let $\Omega=] 0,1[2, N \in \mathbb{N}$, $r=\frac{1}{N}$ and let $|\cdot|$ denote the Euclidean norm into $\mathbb{R}^{3}$. We assume that the lattice nodes coincide at rest with the points $M_{i, j}=(i r, j r) \in \bar{\Omega},(i, j) \in$ $\{0,1, \ldots, N\}^{2}$. For the sake of simplicity, we assume that the lattice is fixed at points located on the boundary. All arguments below extend easily to 
other cases, for instance a lattice whose left edge nodes are fixed. Under a loading $G$ acting on the internal nodes, the lattice undergoes a deformation $\psi$ from $\left\{M_{i, j},(i, j) \in\{0,1, \ldots, N\}^{2}\right\}$ into $\mathbb{R}^{3}$ that minimizes, under the placement constraint, the energy

$$
\begin{aligned}
& \frac{k_{1}}{2} \sum_{0 \leq i \leq N-1,1 \leq j \leq N-1}\left(\left|\psi\left(M_{i+1, j}\right)-\psi\left(M_{i, j}\right)\right|-r\right)^{2} \\
+ & \frac{k_{1}}{2} \sum_{1 \leq i \leq N-1,0 \leq j \leq N-1}\left(\left|\psi\left(M_{i, j+1}\right)-\psi\left(M_{i, j}\right)\right|-r\right)^{2} \\
+ & \frac{k_{2}}{2} \sum_{0 \leq i, j \leq N} \sum_{\left((k, l),\left(k^{\prime}, l^{\prime}\right)\right) \in C_{i j}}\left(\frac{\psi\left(M_{k^{\prime}, l^{\prime}}\right)-\psi\left(M_{i, j}\right)}{\left|\psi\left(M_{k^{\prime}, l^{\prime}}\right)-\psi\left(M_{i, j}\right)\right|} \cdot \frac{\psi\left(M_{k, l}\right)-\psi\left(M_{i, j}\right)}{\left|\psi\left(M_{k, l}\right)-\psi\left(M_{i, j}\right)\right|}\right)^{2} \\
- & \sum_{i, j=1}^{N-1} G\left(M_{i, j}\right) \cdot \psi\left(M_{i, j}\right)
\end{aligned}
$$

where $k_{1}$ and $k_{2}$ are stiffness coefficients and where $C_{i j}$ allows to number the pairs of bars sharing one end identified by $(i, j)$ : for $(i, j) \in\{0,1, \ldots, N-$ $1\}^{2}, C_{i j}$ contains four elements; otherwise it contains two elements. Obviously, in such a model segments between nodes remain straight under any deformation. Therefore, when the deformation of nodes in known, the lattice deformation is known. This is the simplest example of an energy taking into account the length changes and the angle changes due to the deformation. The internal energy is equal to 0 when all segments are of length $r$ and when all angles are equal to $\pi / 2$. Such an energy applies to mechanical networks and to atom lattices as well, in which case segments are atomic bonds. Adjacent nodes cannot be set on a single point because bar lengths cannot be equal to 0 . We do not assume $\psi$ to be globally one-to-one thus allowing for folding along the bars. In other words, $\psi$ must satisfy the constraint $\psi\left(M_{k, l}\right) \neq \psi\left(M_{i, j}\right)$ for adjacent pairs of indices. As a consequence, denominators in (44) are not equal to 0 and the energy is well defined.

We emphasize the fact that the geometry of the lattice we study here is extremely simple: at rest, translating a reference pattern made of one node and two segments along two orthogonal directions generate the whole of the structure. The derivation of a continous model has been performed by formal asymptotic methods in a more general setting. Namely, the reference pattern contains several nodes and an arbitrary number of bars, see Mourad (2003) and Caillerie et al. (2003), and Caillerie et al. (2006) for results specifc to hexagonal networks. Here, we aim at obtaining rigorous convergence proofs and our reasonings are in the spirit of those by Braides and Gelli (2002), Alicandro and Cicalese (2004). 

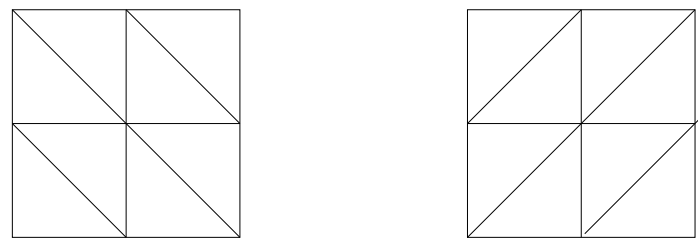

Figure 1. Left: triangulation $\mathcal{T}_{h}^{1}$, right: triangulation $\mathcal{T}_{h}^{2}$

We consider that the square lattice behavior is close to the limit behavior of a sequence of lattices whose grid step $\varepsilon r$ goes to 0 . Data for these lattices are given by assuming that $k_{1}^{\varepsilon}=k_{1}, k_{2}^{\varepsilon}=\varepsilon^{2} k_{2}$ and that there exists a continuous function $g$ defined on $\bar{\Omega}$ such that for all $i, j=1, \ldots, \frac{1}{\varepsilon r}$, $G^{\varepsilon}(i \varepsilon r, j \varepsilon r)=\varepsilon^{2} r^{2} g(i \varepsilon r, j \varepsilon r)$.

In order to write convergence results, we need the minimization problems associated with all lattices in this sequence to be defined on a single functional space. To this aim, we first subdivide squares with edge length $h=\varepsilon r$ into triangles. In other words, we construct a triangulation denoted by $\mathcal{T}_{h}^{1}$ in a way reminiscent of finite element subdivisions (hence the choice of the notation $h$ ), see Fig. 1. With each mapping $\psi$ defined on the nodes $(i h, j h)$ we can associate a mapping defined on $\bar{\Omega}$ that is affine on each triangle, globally continuous and coincides with $\psi$ at each node. This new mapping can still be denoted by $\psi$. Obviously,

$$
\begin{aligned}
& \left.\forall x_{1} \in\right] i h,(i+1) h\left[, \psi((i+1) h, j h)-\psi(i h, j h)=h \partial_{1} \psi\left(x_{1}, j h\right),\right. \\
& \left.\forall x_{2} \in\right] j h,(j+1) h\left[, \psi(i h,(j+1) h)-\psi(i h, j h)=h \partial_{2} \psi\left(i h, x_{2}\right),\right.
\end{aligned}
$$

and $\partial_{1} \psi, \partial_{2} \psi$ are $\mathbb{R}^{3}$-valued functions that remain constant over each triangle. This typically allows to write terms due to the length changes in the energy (44) corresponding to the $h$-lattice as integrals of functions depending on the gradient of $\psi$ only. Inner products in the third line that correspond to two segments belonging to a single triangle of $\mathcal{T}_{h}^{1}$ can be written as integrals of functions depending on the gradient of $\psi$, as well. This trick does not work for inner products corresponding to pairs of bars that are not the edges of a single triangle in $\mathcal{T}_{h}^{1}$. Therefore we introduce a second triangulation denoted by $\mathcal{T}_{h}^{2}$ that is orthogonal to $\mathcal{T}_{h}^{1}$, see Fig. 1 , and with each mapping $\psi$ defined on the nodes $(i h, j h)$ we associate a mapping $\psi^{\perp}$ defined on $\bar{\Omega}$ that is affine on each triangle of $\mathcal{T}_{h}^{2}$, globally continuous and coincides with $\psi$ at each node. Upon some computation and dividing 
by the first stiffness coefficient, we arrive at the family of scaled energies

$$
\begin{aligned}
I_{h}(\psi) & =\int_{\Omega}\left(\left(\left|\partial_{1} \psi\right|-1\right)^{2}+\left(\left|\partial_{2} \psi\right|-1\right)^{2}+K\left(\frac{\partial_{1} \psi}{\left|\partial_{1} \psi\right|} \cdot \frac{\partial_{2} \psi}{\left|\partial_{2} \psi\right|}\right)^{2}\right) d x \\
& +\int_{\Omega}\left(\left(\left|\partial_{1} \psi^{\perp}\right|-1\right)^{2}+\left(\left|\partial_{2} \psi^{\perp}\right|-1\right)^{2}+K\left(\frac{\partial_{1} \psi^{\perp}}{\left|\partial_{1} \psi^{\perp}\right|} \cdot \frac{\partial_{2} \psi^{\perp}}{\left|\partial_{2} \psi^{\perp}\right|}\right)^{2}\right) d x \\
& -L_{h}(\psi)
\end{aligned}
$$

where

$$
K=\frac{4}{r^{2}} \frac{k_{p}}{k_{l}}, L_{h}(\psi)=h^{2} \sum_{i, j=1}^{\frac{1}{h}} f(i h, j h) \cdot \psi(i h, j h) \quad \text { with } \quad f=\frac{4}{k_{l}} g .
$$

defined on the sets

$$
\begin{aligned}
& \mathcal{A}_{h}^{*}=\left\{\psi \in \mathcal{C}^{0}\left(\bar{\Omega} ; \mathbb{R}^{3}\right), \forall T \in \mathcal{T}_{h}^{1}, \psi_{\mid T} \in \mathbb{P}_{1}(T), \psi_{\mid \partial \Omega}=i d,\right. \\
& \left.\forall(k, l),\left(k^{\prime}, l^{\prime}\right) s . t .\left|k^{\prime}-k\right|+\left|l^{\prime}-l\right|=1, \psi\left(k^{\prime} h, l^{\prime} h\right) \neq \psi(k h, l h)\right\} .
\end{aligned}
$$

Functions satisfying the final assertion in (46) will be said to be pairwise one-to-one. In the sequel, we will also use the set $\mathcal{A}_{h}$ which does not require the deformations to be pairwise one-to-one:

$$
\mathcal{A}_{h}=\left\{\psi \in \mathcal{C}^{0}\left(\bar{\Omega} ; \mathbb{R}^{3}\right), \forall T \in \mathcal{T}_{h}^{1}, \psi_{\mid T} \in \mathbb{P}_{1}(T), \psi_{\mid \partial \Omega}=i d\right\} .
$$

\subsection{Convergence results}

Minimizing $I_{h}$ on $\mathcal{A}_{h}^{*}$ amounts to minimize a functional that is defined on a subset of a finite-dimensional space. Nevertheless, the existence of a minimizer is not obvious since $\mathcal{A}_{h}^{*}$ is not closed. Therefore, we concentrate on the limit behavior of almost minimizers. More specifically, we consider functions $\phi_{h}$ such that

$$
\phi_{h} \in \mathcal{A}_{h}^{*}, \forall \psi \in \mathcal{A}_{h}^{*}, I_{h}\left(\phi_{h}\right) \leq I_{h}(\psi)+s(h),
$$

where $s(h) \geq 0, s(h) \rightarrow 0$ when $h \rightarrow 0$. Our arguments follow the lines of reasoning that were described in Section 2. Let us first introduce some notation: let $i d:\left(x_{1}, x_{2}\right) \in \bar{\Omega} \mapsto\left(x_{1}, x_{2}, 0\right) \in \mathbb{R}^{3}$ and let $W_{1}: \mathbb{M}_{3 \times 2} \mapsto \mathbb{R}$ be defined by

$$
\forall F=\left[z_{1} \mid z_{2}\right] \in \mathbb{M}_{3 \times 2}, W_{1}(F)=\left(\left|z_{1}\right|-1\right)^{2}+\left(\left|z_{2}\right|-1\right)^{2} .
$$

This function satisfies the coerciveness inequality

$$
\forall F=\left[z_{1} \mid z_{2}\right] \in \mathbb{M}_{3 \times 2}, W_{1}(F) \geq \frac{1}{2}\|F\|^{2}-1 .
$$


Lemma 3.1. Let $\phi_{h}$ a sequence in $\mathcal{A}_{h}^{*}$ satisfying (48). Then, there exists $C>0$ such that for all $h, I_{h}\left(\phi_{h}\right) \leq C$. Let $\psi_{h} \in \mathcal{A}_{h}^{*}$ be a sequence such that $I_{h}\left(\psi_{h}\right) \leq C<+\infty$ for all $h$. Then $\psi_{h}$ is bounded in $H^{1}\left(\Omega ; \mathbb{R}^{3}\right)$.

Proof- Choosing $\psi=i d$ in (48), we have $\psi^{\perp}=i d$ and we immediately obtain that $I_{h}\left(\phi_{h}\right) \leq I_{h}(i d)+s(h)=-L_{h}(i d)+s(h)$. Since for any $h, L_{h}(i d)$ is a Riemann sum associated with $f \cdot i d$, the sequence $L_{h}(i d)$ converges and we know by construction that $s(h)$ goes to 0 . Therefore, the first point in Lemma 3.1 is proved. Let us turn to the second point. We first establish that

$$
\exists C>0, \forall h, \forall \psi_{h} \in \mathcal{A}_{h},\left(\sum_{h} h^{2}\left|\psi_{h}(i h, j h)\right|^{2}\right)^{1 / 2} \leq\left\|\psi_{h}\right\|_{L^{2}\left(\Omega ; \mathbb{R}^{3}\right)} .
$$

This is obtained by sendind the restrictions of functions $\psi_{h}$ to all triangles in $\mathcal{T}_{h}^{1}$ onto a single triangle, say the unit rectangular triangle $\hat{T}$, as is customary in the finite element theory, and by using the fact that all norms are equivalent on $\mathbb{P}_{1}(\hat{T})$. Now let $\psi_{h} \in \mathcal{A}_{h}^{*}$ be a sequence such that $I_{h}\left(\psi_{h}\right) \leq C<+\infty$. Then, by (51),

$$
\forall h, \int_{\Omega} W_{1}\left(\nabla \psi_{h}(x)\right) d x \leq C+L_{h}\left(\psi_{h}\right) \leq C\left(1+\left\|\psi_{h}\right\|_{L^{2}\left(\Omega ; \mathbb{R}^{3}\right)}\right) .
$$

We conclude by (50) and by Poincaré inequality.

As a consequence, the following corollary holds true.

Corollary 3.2. For any sequence $\phi_{h}$ satisfying (48), there exists a subsequence that we still label by $h$ and there exists $\phi \in H^{1}\left(\Omega ; \mathbb{R}^{3}\right)$ such that $\phi_{h} \rightarrow \phi$ in $L^{2}\left(\Omega ; \mathbb{R}^{3}\right)$ and $\phi_{h} \rightarrow \phi$ in $H^{1}\left(\Omega ; \mathbb{R}^{3}\right)$.

In order to identify a problem whose $\phi$ is a minimizer, we use $\Gamma$-convergence tools. To this end, we extend energies $I_{h}$ to $L^{2}\left(\Omega ; \mathbb{R}^{3}\right)$ in the classical way seen in Section 2. Namely, we set

$$
\forall \psi \in \mathcal{A}_{h}^{*}, \tilde{I}_{h}(\psi)=I_{h}(\psi), \quad \forall \psi \in L^{2}\left(\Omega ; \mathbb{R}^{3}\right) \backslash \mathcal{A}_{h}^{*}, \tilde{I}_{h}(\psi)=+\infty .
$$

Obviously, $\phi_{h}$ solves (48) if and only if $\phi_{h}$ satisfies

$$
\phi_{h} \in L^{2}\left(\Omega ; \mathbb{R}^{3}\right), \forall \psi \in L^{2}\left(\Omega ; \mathbb{R}^{3}\right), \tilde{I}_{h}\left(\phi_{h}\right) \leq \tilde{I}_{h}(\psi)+h s(h) .
$$

Moreover, we need to work on vector spaces rather than on affine spaces. Therefore, we define the sets

$$
\begin{aligned}
\mathcal{V}_{h}^{*}=\mathcal{A}_{h}^{*}-i d= & \left\{v \in \mathcal{C}^{0}\left(\bar{\Omega} ; \mathbb{R}^{3}\right), \forall T \in \mathcal{T}_{h}^{1}, v_{\mid T} \in \mathbb{P}_{1}(T), v_{\mid \partial \Omega}=0,\right. \\
& i d+v \text { pairwise one-to-one }\},
\end{aligned}
$$


with the associated functionals $\tilde{J}_{h}$ given by

$$
\forall v \in \mathcal{V}_{h}^{*}, \tilde{J}_{h}(v)=J_{h}(v):=I_{h}(i d+v), \forall v \in L^{2}\left(\Omega ; \mathbb{R}^{3}\right) \backslash \mathcal{V}_{h}^{*}, \tilde{J}_{h}(v)=+\infty
$$

or equivalently

$$
\forall v \in L^{2}\left(\Omega ; \mathbb{R}^{3}\right), \quad \tilde{J}_{h}(v)=\tilde{I}_{h}(i d+v) .
$$

Finally, we extract a $\Gamma$-convergent subsequence for the $L^{2}\left(\Omega ; \mathbb{R}^{3}\right)$-topology and call $\tilde{J}_{0}$ its $\Gamma$-limit. As usual, the uniqueness of $\tilde{J}_{0}$ will make the extraction of this subsequence superfluous a posteriori.

Proposition 3.3. $u:=\phi-i d$ minimizes $\tilde{J}_{0}$ on $L^{2}\left(\Omega ; \mathbb{R}^{3}\right)$.

Proof- We recall the argument that was already given in Proposition 2.7. From Corollary 3.2, we know that $u_{h}:=\phi_{h}-i d$ converges to $u$ in $L^{2}\left(\Omega ; \mathbb{R}^{3}\right)$. Therefore, on the one hand, $\tilde{J}_{0}(u) \leq \liminf \tilde{J}_{h}\left(u_{h}\right)$. On the other hand, for any $v$ in $L^{2}\left(\Omega ; \mathbb{R}^{3}\right)$, there exists $v_{h}$ in $L^{2}\left(\Omega ; \mathbb{R}^{3}\right)$ such that $\tilde{J}_{h}\left(v_{h}\right)$ converges to $\tilde{J}_{0}(v)$. Therefore, for any $v \in L^{2}\left(\Omega ; \mathbb{R}^{3}\right)$,

$$
\tilde{J}_{0}(u) \leq \liminf \tilde{J}_{h}\left(u_{h}\right) \leq \liminf \left(\tilde{J}_{h}\left(v_{h}\right)+h s(h)\right)=\tilde{J}_{0}(v)
$$

Let us now proceed to the identification of $\tilde{J}_{0}(v)$ for any $v$ in $L^{2}\left(\Omega ; \mathbb{R}^{3}\right)$. The case when $v$ does not belong to $H_{0}^{1}\left(\Omega ; \mathbb{R}^{3}\right)$ is immediate.

Proposition 3.4. For all $v$ in $L^{2}\left(\Omega, \mathbb{R}^{3}\right) \backslash H_{0}^{1}\left(\Omega, \mathbb{R}^{3}\right), \tilde{J}_{0}(v)=+\infty$.

Proof- We proceed by contradiction. Suppose $\tilde{J}_{0}(v)<+\infty$. Since $\tilde{J}_{h}$ $\Gamma$-converges to $\tilde{J}_{0}$ for the $L^{2}\left(\Omega ; \mathbb{R}^{3}\right)$-topology, there exists a sequence $v_{h}$ in $L^{2}\left(\Omega ; \mathbb{R}^{3}\right)$ such that $v_{h} \rightarrow v$ in $L^{2}\left(\Omega ; \mathbb{R}^{3}\right)$ and $\tilde{J}_{h}\left(v_{h}\right) \rightarrow \tilde{J}_{0}(v)<+\infty$. Obviously $\widetilde{J}_{h}\left(v_{h}\right)$ is bounded from above. Therefore, $v_{h}$ belongs to $\mathcal{V}_{h}^{*}$ and from Lemma 3.1, we deduce that $v_{h}$ converges weakly to $v$ in $H^{1}\left(\Omega ; \mathbb{R}^{3}\right)$ which states in particular that $v$ belongs to $H_{0}^{1}\left(\Omega ; \mathbb{R}^{3}\right)$.

This result implies that $u:=\phi-i d$ belongs to $H_{0}^{1}\left(\Omega ; \mathbb{R}^{3}\right)$ and minimizes $\tilde{J}_{0}$ on $H_{0}^{1}\left(\Omega ; \mathbb{R}^{3}\right)$. Before looking for a precise expression of $\tilde{J}_{0}(v)$ when $v$ in $H_{0}^{1}\left(\Omega ; \mathbb{R}^{3}\right)$, let us check that it is a finite number. This step was obvious in the framework of Section 2. Here, it requires some work. Actually, the result will not be obtained by simply defining a constant sequence $v_{h}=v$ and saying that $\tilde{J}_{0}(v) \leq \liminf \tilde{J}_{h}\left(v_{h}\right)=\liminf J_{h}\left(v_{h}\right)<+\infty$. Indeed, $v$ is not necessarily affine not to mention not necessarily such that $i d+v$ is pairwise one-to-one. What we need to do is finding a sequence $v_{h}$ in $\mathcal{V}_{h}^{*}$ such that $v_{h}$ converges to $v$ in $L^{2}\left(\Omega ; \mathbb{R}^{3}\right)$. The following lemma gives stronger 
results. In the first assertion, we ignore the injectivity condition and we make use of the notation

$$
\mathcal{V}_{h}=\mathcal{A}_{h}-i d=\left\{v \in \mathcal{C}^{0}\left(\bar{\Omega} ; \mathbb{R}^{3}\right), \forall T \in \mathcal{T}_{h}^{1}, v_{\mid T} \in \mathbb{P}_{1}(T), v_{\mid \partial \Omega}=0\right\} .
$$

In the second assertion, we take the injectivity condition into account.

Lemma 3.5. For any $v$ in $H_{0}^{1}\left(\Omega ; \mathbb{R}^{3}\right)$, there exists a sequence $v_{h}$ such that $v_{h} \in \mathcal{V}_{h}$ and $v_{h} \rightarrow v$ in $H^{1}\left(\Omega ; \mathbb{R}^{3}\right)$. Moreover elements $v_{h}$ can be taken in $\mathcal{V}_{h}^{*}$.

Proof- Let $\Pi_{h}$ be the $\mathbb{P}_{1}$-interpolation operator associated with $\mathcal{T}_{h}^{1}$. Standard results show that, for any $w$ in $H^{2}\left(\Omega ; \mathbb{R}^{3}\right), \Pi_{h}(w)$ converges to $w$. If $w$ belongs to $\left(H^{2} \cap H_{0}^{1}\right)\left(\Omega ; \mathbb{R}^{3}\right)$, then $\Pi_{h} w$ is equal to 0 on the boundary. In other words, $\Pi_{h} w$ belongs to $\mathcal{A}_{h}$. Therefore, any $w$ in $\left(H^{2} \cap H_{0}^{1}\right)\left(\Omega ; \mathbb{R}^{3}\right)$ is the limit of a sequence $w_{h}, h \rightarrow 0$ with $w_{h}$ in $\mathcal{A}_{h}$. Now, well known density results state that any $v$ in $H_{0}^{1}\left(\Omega ; \mathbb{R}^{3}\right)$ is the limit of a sequence $w_{n}$, $n \rightarrow+\infty$ such that $w_{n}$ belongs to $\left(H^{2} \cap H_{0}^{1}\right)\left(\Omega ; \mathbb{R}^{3}\right)$. Upon extracting a diagonal sequence (recall that $h$ is the inverse of an integer), we prove the first assertion of Lemma 3.5.

For obtaining the second assertion, we show that functions $v_{h}$ that were just obtained can be slightly modified into functions $v_{h}^{*}$ such that the functions $\psi_{h}^{*}:=\left(i d+v_{h}^{*}\right)$ are pairwise one-to-one. We actually prove a stronger result, since we obtain functions $\psi_{h}^{*}$ whose restrictions to the set of nodes are one-to-one. Define $\psi_{h}=i d+v_{h}$ and

$$
N_{h}=\left\{\left((k, l),\left(k^{\prime}, l^{\prime}\right)\right), k, l, k^{\prime}, l^{\prime} \in\left\{0, \ldots, \frac{1}{h}\right\}^{2} ; \psi_{h}(k h, l h) \neq \psi_{h}\left(k^{\prime} h, l^{\prime} h\right)\right\} .
$$

This set is nonempty since $\psi_{h}=i d$ on the boundary. Therefore, $C_{h}:=$ $\min _{N_{h}} \mid\left(\psi_{h}\left(k^{\prime} h, l^{\prime} h\right)-\psi(k h, l h) \mid\right.$ is strictly positive. Finally, let $\lambda_{h}=\frac{C_{h}}{2 \sqrt{2}}$. We define $v_{h}^{*}$ as follows:

$$
\forall\left(x_{1}, x_{2}\right) \text { s.t. } h \leq x_{1}, x_{2} \leq 1-h, v_{h}^{*}=v_{h}+\lambda_{h} i d, v_{h \mid \partial \Omega}^{*}=0
$$

and $v_{h}^{*}$ is globally continuous and affine on each triangle in $\mathcal{T}_{h}^{1}$. Note that since $v_{h}$ and $i d$ are affine per triangle, there is no contradiction in the previous requirements. Let us check that the restriction of $\psi_{h}^{*}=i d+v_{h}^{*}$ to the set of nodes is one-to-one. Suppose that $\psi_{h}^{*}(k h, l h)=\psi_{h}^{*}\left(k^{\prime} h, l^{\prime} h\right)$ with $(k, l),\left(k^{\prime}, l^{\prime}\right) \in\left\{0, \ldots, \frac{1}{h}\right\}^{2}$. Let us show that $(k, l)=\left(k^{\prime}, l^{\prime}\right)$. We distinguish several cases:

- Case 1: $(k h, l h)$ and $\left(k^{\prime} h, l^{\prime} h\right)$ belong to $\partial \Omega$. Since $\psi_{h}$ coincides with $i d$ on $\partial \Omega$, obviously $(k, l)=\left(k^{\prime}, l^{\prime}\right)$. 
- Case 2: $(k h, l h)$ and $\left(k^{\prime} h, l^{\prime} h\right)$ belong to $\Omega$. Then

- either $\psi_{h}(k h, l h)=\psi_{h}\left(k^{\prime} h, l^{\prime} h\right)$ and the assumption on $\psi_{h}^{*}$ implies that $\lambda_{h}(k h, l h, 0)=\lambda_{h}\left(k^{\prime} h, l^{\prime} h, 0\right)$, whence $(k, l)=\left(k^{\prime}, l^{\prime}\right)$ because $\lambda_{h} \neq 0$,

- or $\psi_{h}(k h, l h) \neq \psi_{h}\left(k^{\prime} h, l^{\prime} h\right)$ and the assumption on $\psi_{h}^{*}$ implies that $\psi_{h}(k h, l h)-\psi_{h}\left(k^{\prime} h, l^{\prime} h\right)=\lambda_{h}\left(\left(k^{\prime}-k\right) h,\left(l^{\prime}-l\right) h, 0\right)$. Therefore, $\mid \psi_{h}(k h, l h)-$ $\psi_{h}\left(k^{\prime} h, l^{\prime} h\right) \mid \leq \lambda_{h} \sqrt{2}<\frac{C_{h}}{2}$ which is contradictory with the definition of $C_{h}$. - Case 3: $(k h, l h) \in \partial \Omega$ and $\left(k^{\prime} h, l^{\prime} h\right) \in \Omega$. Similar to case 2 .

We still have to prove that $v_{h}^{*}$ converges to $v$ in $H^{1}\left(\Omega ; \mathbb{R}^{3}\right)$. Clearly, $C_{h} \leq h$ and $\lambda_{h} \leq \frac{h}{2 \sqrt{2}}$. Therefore, $v_{h}+\lambda_{h} i d$ converges to $v$ in $H^{1}\left(\Omega ; \mathbb{R}^{3}\right)$. The result remains true for $v_{h}^{*}$ which differs from $v_{h}+\lambda_{h} i d$ on bands of width $h: L^{\infty}$ bounds for $v_{h}^{*}$ and its partial derivatives on these bands are easily obtained.

Corollary 3.6. $\tilde{J}_{0}$ is finite on $H_{0}^{1}\left(\Omega ; \mathbb{R}^{3}\right)$.

Proof- For any $v$ in $H_{0}^{1}\left(\Omega ; \mathbb{R}^{3}\right)$, there exists $v_{h}$ in $\mathcal{V}_{h}^{*}$ such that $v_{h} \rightarrow v$ in $L^{2}\left(\Omega ; \mathbb{R}^{3}\right)$. Therefore,

$$
\tilde{J}_{0}(v) \leq \liminf \tilde{J}_{h}\left(v_{h}\right)=\liminf J_{h}\left(v_{h}\right)<+\infty .
$$

Finding an expression for $\tilde{J}_{0}(v), v$ in $H_{0}^{1}\left(\Omega ; \mathbb{R}^{3}\right)$ is now divided into two steps. First, we exhibit a functional that is smaller than $\tilde{J}_{0}$; then, we prove that it is greater as well. Let us recall the definition of $J_{h}$. For any $v_{h}$ in $\mathcal{V}_{h}^{*}$ and $\psi_{h}=i d+v_{h}$,

$J_{h}\left(v_{h}\right)=I_{h}\left(\psi_{h}\right)=\int_{\Omega}\left(W_{1}+W_{2}\right)\left(\nabla \psi_{h}(x)\right)+\left(W_{1}+W_{2}\right)\left(\nabla \psi_{h}^{\perp}(x)\right) d x-L_{h}\left(\psi_{h}\right)$,

where

$$
\forall F=\left[z_{1} \mid z_{2}\right] \in \mathbb{M}_{3 \times 2} \text { s.t. } z_{1} \neq 0 \text { and } z_{2} \neq 0, W_{2}(F)=K\left(\frac{z_{1}}{\left|z_{1}\right|} \cdot \frac{z_{2}}{\left|z_{2}\right|}\right)^{2} .
$$

Let $v$ in $H_{0}^{1}\left(\Omega ; \mathbb{R}^{3}\right)$. By the definition of $\Gamma$-convergence, there exists $v_{h}$ in $L^{2}\left(\Omega ; \mathbb{R}^{3}\right)$ such that $v_{h} \rightarrow v$ in $L^{2}\left(\Omega ; \mathbb{R}^{3}\right)$ and $\tilde{J}_{h}\left(v_{h}\right) \rightarrow \tilde{J}_{0}(v)<+\infty$. Equivalently, $\psi_{h}=i d+v_{h}$ converges to $\psi=i d+v$ in $L^{2}\left(\Omega ; \mathbb{R}^{3}\right)$ and $\tilde{I}_{h}\left(\psi_{h}\right)$ converges to $\tilde{I}_{0}(\psi)<+\infty$. From Lemma 3.1 , we derive that $\psi_{h}$ belongs to $\mathcal{A}_{h}^{*}$ and converges weakly to $\psi$ in $H^{1}\left(\Omega ; \mathbb{R}^{3}\right)$. Let us show that $\psi_{h}^{\perp}$ exhibits similar properties.

Lemma 3.7. For any sequence $\psi_{h}$ in $\mathcal{A}_{h}$ such that $\psi_{h}$ converges to $\psi$ strongly in $L^{2}\left(\Omega ; \mathbb{R}^{3}\right)$ and weakly in $H^{1}\left(\Omega ; \mathbb{R}^{3}\right)$, the sequence $\psi_{h}^{\perp}$ converges to $\psi$ strongly in $L^{2}\left(\Omega ; \mathbb{R}^{3}\right)$ and weakly in $H^{1}\left(\Omega ; \mathbb{R}^{3}\right)$ as well. Moreover, $\left\|\nabla \psi_{h}^{\perp}\right\|_{L^{2}\left(\Omega ; \mathbb{M}_{3 \times 2}\right)}=\left\|\nabla \psi_{h}\right\|_{L^{2}\left(\Omega ; \mathbb{M}_{3 \times 2}\right)}$. 
Proof- Let $Q$ be the square with vertices $(i h, j h),((i+1) h, j h),((i+$ 1) $h,(j+1) h),(i h,(j+1) h)$. We divide $Q$ into two triangles $T_{1}$ and $T_{3}$ where the vertices of $T_{1}$ (resp. $\left.T_{3}\right)$ are $(i h, j h),((i+1) h, j h),(i h,(j+1) h)$ (resp. $((i+1) h, j h),((i+1) h,(j+1) h),(i h,(j+1) h)$. We divide $Q$ into two triangles $T_{2}$ and $T_{4}$ as well where the vertices of $T_{2}$ (resp. $\left.T_{4}\right)$ are $(i h, j h),((i+$ 1) $h, j h),((i+1) h,(j+1) h)($ resp. $(i h, j h),((i+1) h,(j+1) h),(i h,(j+1) h)$. Restricted to $T_{1}$ (resp. $T_{3}$ ), $\partial_{1} \psi_{h}$ is a constant vector that is equal to $\partial_{1} \psi_{h}^{\perp}$ restricted to $T_{2}$ (resp. $T_{4}$ ). Therefore,

$$
\int_{Q}\left|\partial_{1} \psi_{h}\right|^{2} d x=\int_{T_{1} \cup T_{3}}\left|\partial_{1} \psi_{h}\right|^{2} d x=\int_{T_{2} \cup T_{4}}\left|\partial_{1} \psi_{h}^{\perp}\right|^{2} d x=\int_{Q}\left|\partial_{1} \psi_{h}^{\perp}\right|^{2} d x .
$$

Similar equalities hold for the derivatives with respect to $x_{2}$. Upon adding the equalities for all squares $Q$, we obtain

$$
\left\|\nabla \psi_{h}^{\perp}\right\|_{L^{2}\left(\Omega ; \mathbb{M}_{3 \times 2}\right)}=\left\|\nabla \psi_{h}\right\|_{L^{2}\left(\Omega ; \mathbb{M}_{3 \times 2}\right)} .
$$

Hence, $\left\|\nabla \psi_{h}^{\perp}\right\|_{L^{2}\left(\Omega ; \mathbb{M}_{3 \times 2}\right)}$ is bounded. As $\psi_{h}^{\perp}$ coincides with the identity on $\partial \Omega$, Poincaré inequality proves that $\psi_{h}^{\perp}$ is bounded in $H^{1}\left(\Omega ; \mathbb{R}^{3}\right)$. Therefore $\psi_{h}^{\perp}$ converges weakly to some function in $H_{0}^{1}\left(\Omega ; \mathbb{R}^{3}\right)$. Let us now prove that $\chi_{h}:=\psi_{h}^{\perp}-\psi_{h}$ converges to 0 in $L^{2}\left(\Omega ; \mathbb{R}^{3}\right)$. Since $\psi_{h}$ and $\psi_{h}^{\perp}$ coincide on the vertices on any $Q$ defined above, they coincide on the edges of $Q$. In other words, $\chi_{h}$ is equal to 0 on $\partial Q$, and rewriting the classical proof of Poincaré inequality reads

$$
\chi_{h}\left(x_{1}, x_{2}\right)=\int_{i h}^{x_{1}} \partial_{1} \chi_{h}\left(\xi_{1}, x_{2}\right) d \xi_{1},
$$

which provides by Cauchy-Schwarz inequality,

$$
\left|\chi_{h}\left(x_{1}, x_{2}\right)\right|^{2} \leq h \int_{i h}^{(i+1) h}\left(\partial_{1} \chi_{h}\right)^{2}\left(\xi_{1}, x_{2}\right) d \xi_{1},
$$

and upon integrating with respect to $x_{2}$

$$
\int_{j h}^{(j+1) h}\left|\chi_{h}\left(x_{1}, x_{2}\right)\right|^{2} d x_{2} \leq h \int_{Q}\left|\partial_{1} \chi_{h}(x)\right|^{2} d x .
$$

Finally

$$
\int_{Q}\left|\chi_{h}(x)\right|^{2} d x \leq h^{2} \int_{Q}\left|\partial_{1} \chi_{h}(x)\right|^{2} d x, \int_{\Omega}\left|\chi_{h}(x)\right|^{2} d x \leq h^{2} \int_{\Omega}\left|\partial_{1} \chi_{h}(x)\right|^{2} d x,
$$

which implies that $\left\|\chi_{h}\right\|_{L^{2}\left(\Omega ; \mathbb{R}^{3}\right.} \leq h\left\|\nabla \chi_{h}\right\|_{L^{2}\left(\Omega ; \mathbb{M}_{3 \times 2}\right)}$. Using the first part of the proof, we obtain that $\psi_{h}^{\perp}-\psi_{h}$ converges to 0 in $L^{2}\left(\Omega ; \mathbb{R}^{3}\right)$ from which Lemma 3.7 follows. 
Let us now proceed to study the limit behavior of $I_{h}\left(\psi_{h}\right)$. To this aim, we extend $W_{2}$ to the whole of $\mathbb{M}_{3 \times 2}$ by letting: for all $F=\left[z_{1} \mid z_{2}\right]$ in $\mathbb{M}_{3 \times 2}$,

$$
W_{2}^{e}(F)=W_{2}(F) \text { if } z_{1} \neq 0 \text { and } z_{2} \neq 0, W_{2}^{e}(F)=K \text { if not. }
$$

This new function $W_{2}^{e}$ is not continuous on $\mathbb{M}_{3 \times 2}$, but it is Borel measurable and bounded by $K$. As a consequence, $Z$ defined by

$$
Z: \mathbb{M}_{3 \times 2} \mapsto \mathbb{R}, Z=W_{1}+W_{2}^{e}
$$

is Borel measurable and satisfies the coerciveness and growth inequalities

$$
\forall F \in \mathbb{M}_{3 \times 2}, \alpha\|F\|^{2}-\beta \leq Z(F) \leq \alpha^{\prime}\|F\|^{2}+\beta^{\prime}, \alpha, \alpha^{\prime}, \beta, \beta^{\prime}>0 .
$$

Since $W_{2}^{e}$ extends $W_{2}$, we can write, see (59),

$$
I_{h}\left(\psi_{h}\right)=\int_{\Omega}\left(Z\left(\nabla \psi_{h}(x)\right)+Z\left(\nabla \psi_{h}^{\perp}(x)\right)\right) d x-L_{h}\left(\psi_{h}\right) .
$$

Let $Q Z$ be the quasiconvex envelope of $Z$ which is classically defined, see Dacorogna (2007), by

$$
Q Z(F)=\sup \left\{g(F) ; g: \mathbb{M}_{3 \times 2} \mapsto \mathbb{R}, g \text { quasiconvex, } g \leq Z\right\} .
$$

Since $Z$ takes finite values only, all functions $g$ in (65) are continuous: indeed, rank-one convex functions that are finite valued are continuous. Therefore, $Q Z$ is upper semicontinuous, hence Borel measurable and quasiconvex. Let us note finally that

$$
\forall F \in \mathbb{M}_{3 \times 2}, 0 \leq Q Z(F) \leq \alpha^{\prime}\|F\|^{2}+\beta^{\prime},
$$

and let us define $W$ on $\mathbb{M}_{3 \times 2}$ by

$$
W=2 Z=2\left(W_{1}+W_{2}^{e}\right) .
$$

Proposition 3.8. For all $\psi$ in $\left(i d+H_{0}^{1}\left(\Omega ; \mathbb{R}^{3}\right)\right), \tilde{I}_{0}(\psi) \geq \int_{\Omega} Q W(\nabla \psi(x)) d x-$ $\int_{\Omega} f(x) \cdot \psi(x) d x$.

Proving this proposition requires to deal in particular with the term due to the external loadings. As this is not central to our analysis, we do not detail the proof of the following lemma although it is not a totally trivial exercise in approximation theory.

Lemma 3.9. For any sequence $\psi_{h}$ in $\mathcal{A}_{h}$ such that $\psi_{h}$ converges weakly to $\psi$ in $H^{1}\left(\Omega, \mathbb{R}^{3}\right), L_{h}\left(\psi_{h}\right)$ converges to $\int_{\Omega} f(x) \cdot \psi(x) d x$. 
Proof of Proposition 3.8 - Obviously,

$$
I_{h}\left(\psi_{h}\right) \geq \int_{\Omega}\left(Q Z\left(\nabla \psi_{h}(x)\right)+Q Z\left(\nabla \psi_{h}^{\perp}(x)\right)\right) d x-L_{h}\left(\psi_{h}\right),
$$

where both integrands are in $L^{1}(\Omega ; \mathbb{R})$ since $Q Z$ is continuous and satisfies (66). For the same reasons, the functional

$$
H: \psi \in H^{1}\left(\Omega ; \mathbb{R}^{3}\right) \mapsto H(\psi)=\int_{\Omega} Q Z(\nabla \psi(x)) d x \in \mathbb{R}
$$

is well defined. It has been proved by Acerbi and Fusco (1984), see also Dacorogna (2007), that under properties (66), $H$ is sequentially weakly lower semicontinuous on $H^{1}\left(\Omega ; \mathbb{R}^{3}\right)$. Therefore,

$$
\begin{aligned}
\tilde{I}_{0}(\psi)=\lim I_{h}\left(\psi_{h}\right) & \geq \liminf \left(H\left(\psi_{h}\right)+H\left(\psi_{h}^{\perp}\right)\right)-\lim L_{h}\left(\psi_{h}\right) \\
& \geq \liminf H\left(\psi_{h}\right)+\liminf H\left(\psi_{h}^{\perp}\right)-\int_{\Omega} f(x) \cdot \psi(x) d x \\
& \geq 2 H(\psi)-\int_{\Omega} f(x) \cdot \psi(x) d x,
\end{aligned}
$$

since by Lemma 3.7 both sequences $\psi_{h}$ and $\psi_{h}^{\perp}$ converge weakly to $\psi$.

The remaining part of this section is devoted to showing that the inequality in Proposition 3.8 is actually an identity.

Proposition 3.10. For all $\psi$ in $\left(i d+H_{0}^{1}\left(\Omega ; \mathbb{R}^{3}\right)\right), \tilde{I}_{0}(\psi) \leq \int_{\Omega} Q W(\nabla \psi(x)) d x-$ $\int_{\Omega} f(x) \psi(x) d x$.

Proof - By the definition of $\Gamma$-convergence, $\tilde{I}_{0}(\psi)=\tilde{J}_{0}(v) \leq \liminf I_{h}\left(\psi_{h}\right)$ for any sequence $\psi_{h} \in \mathcal{A}_{h}^{*}$ that converges to $\psi$ in $L^{2}\left(\Omega ; \mathbb{R}^{3}\right)$. From Lemma 3.5 , we can choose a sequence $\psi_{h} \in \mathcal{A}_{h}^{*}$ that converges to $\psi$ in $H^{1}\left(\Omega ; \mathbb{R}^{3}\right)$. Let us check that $\psi_{h}^{\perp}$ converges to $\psi$ in $H^{1}\left(\Omega ; \mathbb{R}^{3}\right)$ as well. From Lemma 3.7, we already know that $\psi_{h}^{\perp}$ converges to $\psi$ strongly in $L^{2}\left(\Omega ; \mathbb{R}^{3}\right)$ and weakly in $H^{1}\left(\Omega ; \mathbb{R}^{3}\right)$. Therefore, it suffices to show that $\left\|\psi_{h}^{\perp}\right\|_{H^{1}\left(\Omega ; \mathbb{R}^{3}\right)} \rightarrow\|\psi\|_{H^{1}\left(\Omega ; \mathbb{R}^{3}\right)}$. Actually, from Lemma 3.5 again,

$$
\begin{aligned}
\left\|\psi_{h}^{\perp}\right\|_{H^{1}\left(\Omega ; \mathbb{R}^{3}\right)} & =\left\|\psi_{h}^{\perp}\right\|_{L^{2}\left(\Omega ; \mathbb{R}^{3}\right)}+\left\|\nabla \psi_{h}^{\perp}\right\|_{L^{2}\left(\Omega ; \mathbb{M}_{3 \times 2}\right)} \\
& =\left\|\psi_{h}^{\perp}\right\|_{L^{2}\left(\Omega ; \mathbb{R}^{3}\right)}+\left\|\nabla \psi_{h}\right\|_{L^{2}\left(\Omega ; \mathbb{M}_{3 \times 2}\right)} .
\end{aligned}
$$

Hence,

$$
\left\|\psi_{h}^{\perp}\right\|_{H^{1}\left(\Omega ; \mathbb{R}^{3}\right)} \rightarrow\|\psi\|_{L^{2}\left(\Omega ; \mathbb{R}^{3}\right)}+\|\nabla \psi\|_{L^{2}\left(\Omega ; \mathbb{M}_{3 \times 2}\right)}=\|\psi\|_{H^{1}\left(\Omega ; \mathbb{R}^{3}\right)} .
$$


Let us examine the limit behavior of

$$
I_{h}\left(\psi_{h}\right)=\int_{\Omega}\left(Z\left(\nabla \psi_{h}(x)\right)+Z\left(\nabla \psi_{h}^{\perp}(x)\right)\right) d x-L_{h}\left(\psi_{h}\right)
$$

where we know that $\psi_{h} \rightarrow \psi, \psi_{h}^{\perp} \rightarrow \psi$ in $H^{1}\left(\Omega ; \mathbb{R}^{3}\right)$, and where we recall that $Z$ is not continuous on the set of matrices whose a column is equal to 0 . We separate $I_{h}\left(\psi_{h}\right)$ in two parts by writing $I_{h}\left(\psi_{h}\right)=X_{h}+Y_{h}$, with

$$
\begin{aligned}
X_{h} & =\int_{\Omega}\left(W_{1}\left(\nabla \psi_{h}(x)\right)+W_{1}\left(\nabla \psi_{h}^{\perp}(x)\right)\right) d x-L_{h}\left(\psi_{h}\right), \\
Y_{h} & =\int_{\Omega}\left(W_{2}\left(\nabla \psi_{h}(x)\right)+W_{2}\left(\nabla \psi_{h}^{\perp}(x)\right)\right) d x .
\end{aligned}
$$

Since $W_{1}$ is continuous on $\mathbb{M}_{3 \times 2}$, by the dominated convergence theorem and by Lemma 3.9 ,

$$
X_{h} \rightarrow \int_{\Omega} 2 W_{1}(\nabla \psi(x)) d x-\int_{\Omega} f(x) \cdot \psi(x) d x .
$$

In order to deal with $Y_{h}$, we choose an element $\delta_{1}$ (resp. $\delta_{2}$ ) in the $\mathcal{L}^{2}$ class of $\partial_{1} \psi$ (resp. $\partial_{2} \psi$ ) and we decompose $\Omega$ in two measurable subsets defined by

$$
\Omega_{1}=\left\{x \in \Omega ; \delta_{1}(x) \neq 0 \text { and } \delta_{2}(x) \neq 0\right\}, \Omega_{2}=\Omega \backslash \Omega_{1} .
$$

Clearly,

$$
\begin{gathered}
Y_{h}=\int_{\Omega_{1}}\left(W_{2}\left(\nabla \psi_{h}(x)\right)+W_{2}\left(\nabla \psi_{h}^{\perp}(x)\right) d x\right. \\
+\quad \int_{\Omega_{2}}\left(W_{2}\left(\nabla \psi_{h}(x)\right)+W_{2}\left(\nabla \psi_{h}^{\perp}(x)\right) d x .\right.
\end{gathered}
$$

The dominated convergence theorem allows to deal with the first term and we get

$$
\begin{aligned}
\int_{\Omega_{1}}\left(W_{2}\left(\nabla \psi_{h}(x)\right)+W_{2}\left(\nabla \psi_{h}^{\perp}(x)\right) d x \rightarrow \quad\right. & 2 \int_{\Omega_{1}} W_{2}(\nabla \psi(x)) d x \\
& =2 \int_{\Omega_{1}} W_{2}^{e}(\nabla \psi(x)) d x .
\end{aligned}
$$

For the second term we simply remark that

$$
\int_{\Omega_{2}}\left(W_{2}\left(\nabla \psi_{h}(x)\right)+W_{2}\left(\nabla \psi_{h}^{\perp}(x)\right) d x \leq 2 K \operatorname{meas}\left(\Omega_{2}\right)=2 \int_{\Omega_{2}} W_{2}^{e}(\nabla \psi(x)) d x .\right.
$$


Therefore,

$$
\liminf Y_{h} \leq 2 \int_{\Omega} W_{2}^{e}(\nabla \psi(x)) d x
$$

At this point, we can say that

$$
\forall \psi \in\left(i d+H_{0}^{1}\left(\Omega ; \mathbb{R}^{3}\right)\right), \tilde{I}_{0}(\psi) \leq \int_{\Omega} 2 Z(\nabla \psi(x)) d x-\int_{\Omega} f(x) \cdot \psi(x) d x,
$$

or equivalently

$$
\forall v \in H_{0}^{1}\left(\Omega ; \mathbb{R}^{3}\right), \tilde{J}_{0}(v) \leq G(v),
$$

where $G(v)=2 \int_{\Omega} Z(\nabla \psi(x)) d x-\int_{\Omega} f(x) \cdot \psi(x) d x$. Since $\tilde{J}_{0}$ is sequentially weakly lower semicontinuous on $H_{0}^{1}\left(\Omega ; \mathbb{R}^{3}\right)$, it follows that $\tilde{J}_{0} \leq \Gamma-G$ where $\Gamma-G$ is the sequential weak lower semicontinuous envelope of $G$ on $H_{0}^{1}\left(\Omega ; \mathbb{R}^{3}\right)$. We made use several times in this manuscript of the fact for $Z: \mathbb{M}_{3 \times 2} \mapsto \mathbb{R}$ continuous, positive and satisfying coerciveness and growth hypotheses (63), the sequential weak lower semicontinuous envelope $\Gamma-F$ of $F$ defined by $F(v)=\int_{\Omega} Z(\nabla v(x)) d x$ is given by $\Gamma-F(v)=$ $\int_{\Omega} Q Z(\nabla v(x)) d x$. Although less known, the result remains true when $Z$ is no longer continuous, but Borel measurable, see the relaxation theorems proved in Dacorogna (2007). This ends the proof of Proposition 3.10.

To conclude this section, we can state our final theorem.

Theorem 3.11. For all $\psi$ in $\left(i d+H_{0}^{1}\left(\Omega ; \mathbb{R}^{3}\right)\right), \tilde{I}_{0}(\psi)=\int_{\Omega} Q W(\nabla \psi(x)) d x-$ $\int_{\Omega} f(x) \cdot \psi(x) d x$.

Comments. The homogenized lattice energy $Q W$ inherits the invariance properties of $W$. On the one hand, $W$ is obviously frame indifferent and it is immediate that $Q W$ is frame indifferent as well. Therefore, $Q W$ can be expressed as a function of $C=F^{T} F$; in other words, with obvious notation, there exists a function $w:\left\{\left(c_{11}, c_{22}, c_{12}\right) \in \mathbb{R}^{3} ; c_{11} \geq 0, c_{12}^{2} \leq c_{11} c_{22}\right\}$ such that for all $F$ in $\mathbb{M}_{3 \times 2}, W(F)=w\left(c_{11}, c_{22}, c_{12}\right)$. On the other hand, the planar rotation of angle $\pi / 2$ belongs to the symmetry group of $W$ and this remains true for $Q W$. As a consequence, it is easily shown that $w$ is symmetric with respect to its first two variables. For more general results on the invariance of envelopes, see Bousselsal and Le Dret (2002). Finally, let us mention that $Q W(F)=0$ for matrices $F$ such that $c_{11} \leq 1, c_{22} \leq 1$, $c_{12}^{2} \leq\left(1-c_{11}\right)\left(1-c_{22}\right)$. Therefore, $Q W$ does not coincide with $W$.

\section{Bibliography}

E. Acerbi and N. Fusco. Semicontinuity problems in the calculus of variations. Arch. Rational Mech. Anal., 86:125-145, 1984. 
E. Acerbi, G. Buttazzo, and D. Percivale. A variational definition for the strain energy of an elastic string. J. Elasticity, 25:137-148, 1991.

R. Alicandro and M. Cicalese. A general integral representation result for continuum limits of discrete energies with superlinear growth. SIAM J. Math. Anal., 36:1-37, 2004.

O. Anza Hafsa and J.-Ph. Mandallena. The nonlinear membrane model: Variational derivation under the constraint $\operatorname{det} \nabla u \neq 0$. J. Maths Pures Appl., 86:100-115, 2006.

E. Attouch. Variational Convergence for Functions and Operators. Pitman, 1984.

J.M. Ball. Convexity conditions and existence theorems in nonlinear elasticity. Arch. Rational Mech. Anal., 63:337-403, 1977.

M. Bousselsal and H. Le Dret. Relaxation of functionals involving homogeneous functions and invariance of envelopes. Chin. Ann. of Math., 23: 37-53, 2002.

A. Braides and M.S. Gelli. Continuum limits of discrete sytems without convexity hypotheses. Math. Mech. Solids, 7:41-66, 2002.

D. Caillerie, A. Mourad, and A. Raoult. Cell-to-muscle homogenization. Application to a constitutive law for the myocardium. Math. Model. Num. Anal., 37:681-698, 2003.

D. Caillerie, A. Mourad, and A. Raoult. Discrete homogenization in graphene sheet modeling. J. Elas., 84:33-68, 2006.

P.G. Ciarlet. Mathematical Elasticity. Vol I: Three-dimensional Elasticity. North-Holland, 1987.

P.G. Ciarlet and Ph. Destuynder. A justification of the two-dimensional plate structure. J. Mécanique, 18:315-344, 1979.

B. Dacorogna. Direct Methods in the Calculus of Variations, 2nd edition. Springer, 2007.

G. Dal Maso. An Introduction to $\Gamma$-convergence. Birkäuser, 1993.

E. De Giorgi and T. Franzoni. Su un tipo di convergenza variazionale. Atti. Accad. Naz. Lincei, 58:842-850, 1975.

I. Ekeland and R. Temam. Analyse Convexe et Problèmes Variationnels. Dunod, 1974.

I. Fonseca and S. Müller. A-quasiconvexity, lower semicontinuity and Young measures. SIAM J. Math. Anal., 30:1355-1390, 1999.

D. Fox, A. Raoult, and J.C. Simo. A justification of nonlinear properly invariant plate theories. Arch. Rational Mech. Anal., 124:157-199, 1993.

E. Friesecke, R.D. James, and S. Müller. A theorem on geometric rigidity and the derivation of nonlinear plate theory from three dimensional elasticity. Comm. Pure Appl. Math., 55:1461-1506, 2002. 
E. Friesecke, R.D. James, and S. Müller. A hierarchy of plate models derived from nonlinear elasticity by $\Gamma$-convergence. Arch. Rational Mech. Anal., 180:183-236, 2006.

H. Le Dret. Sur les fonctions de matrices convexes et isotropes. C.R. Acad. Sci. Paris, I, 310:617-620, 1990.

H. Le Dret and A. Raoult. Variational convergence for nonlinear shell models with directors and related semicontinuity and relaxation results. Arch. Rational Mech. Anal., 154:101-134, 2000.

H. Le Dret and A. Raoult. Remarks on the quasiconvex envelope of stored energy functions in nonlinear elasticity. Comm. Applied Nonlinear An., 1:85-96, 1994.

H. Le Dret and A. Raoult. The nonlinear membrane model as variational limit of nonlinear three-dimensional elasticity. J. Math. Pures Appl., 75: 551-580, 1995a.

H. Le Dret and A. Raoult. The quasiconvex envelope of the Saint VenantKirchhoff stored energy function. Proc. Roy. Soc. Edinburgh, 125A:11791192, 1995b.

H. Le Dret and A. Raoult. Quasiconvex envelopes of stored energy densities that are convex with respect to the strain tensor. In C. Bandle, J. Bemelmans, M. Chipot, J. Saint Jean Paulin, and I. Shafrir, editors, Progress in Partial Differential Equations, Pont-à-Mousson, 1994. Pitman, 1995c.

C.B. Morrey. Quasiconvexity and the semicontinuity of multiple integrals. Pacific J. Math., 2:25-53, 1995.

A. Mourad. Description Topologique de l'Architecture Fibreuse et Modélisation Mécanique du Myocarde. Doctoral dissertation, Institut National Polytechnique de Grenoble, 2003.

O. Pantz. Quelques Problèmes de Modélisation en Élasticité non Linéaire. Doctoral dissertation, Université Pierre et Marie Curie, 2001.

A.C. Pipkin. Convexity conditions for strain-dependent energy functions for membranes. Arch. Rational Mech. Anal., 121:361-376, 1993.

A.C. Pipkin. Relaxed energies for large deformations of membranes. IMA J. Appl. Math., 52:297-308, 1994.

A. Raoult. Non-polyconvexity of the stored energy function of a Saint Venant-Kirchhoff material. Aplikace Matematiky, 31:417-419, 1986.

V. Sverak. Rank-one convexity does not imply quasiconvexity. Proc. Roy. Soc. Edinburgh, 120A:185-189, 1992.

R.C. Thompson and L.J. Freede. Eigenvalues of sums of hermitian matrices. J. Research Nat. Bur. Standards B, 75B:115-120, 1971. 